\title{
Dynamic Modeling and Nonlinear Position Control of a Quadruped Robot with Theo Jansen Linkage Mechanisms and a Single Actuator
}

\author{
Shunsuke Nansai, ${ }^{1}$ Rajesh Elara Mohan, ${ }^{2}$ Ning Tan, ${ }^{2}$ Nicolas Rojas, ${ }^{3}$ and Masami Iwase ${ }^{1}$ \\ ${ }^{1}$ Tokyo Denki University, 5 Senjuasahicho, Adachi, Tokyo 120-8551, Japan \\ ${ }^{2}$ Singapore University of Technology and Design, 8 Somapah Road, Singapore 487372 \\ ${ }^{3}$ Yale University, New Haven, CT 06520, USA \\ Correspondence should be addressed to Ning Tan; ning_tan@sutd.edu.sg
}

Received 10 February 2015; Accepted 15 April 2015

Academic Editor: Shahram Payandeh

Copyright ( 2015 Shunsuke Nansai et al. This is an open access article distributed under the Creative Commons Attribution License, which permits unrestricted use, distribution, and reproduction in any medium, provided the original work is properly cited.

The Theo Jansen mechanism is gaining widespread popularity among the legged robotics community due to its scalable design, energy efficiency, low payload-to-machine-load ratio, bioinspired locomotion, and deterministic foot trajectory. In this paper, we perform for the first time the dynamic modeling and analysis on a four-legged robot driven by a single actuator and composed of Theo Jansen mechanisms. The projection method is applied to derive the equations of motion of this complex mechanical system and a position control strategy based on energy is proposed. Numerical simulations validate the efficacy of the designed controller, thus setting a theoretical basis for further investigations on Theo Jansen based quadruped robots.

\section{Introduction}

Legged robots [1-3] have always been a popular choice for robotic researchers because of their superiority over traditional wheeled or tracked robotic platforms on applications involving maneuverability over rough terrains. Bartsch et al. [4] presented their efforts in developing a six-legged, bioinspired, and energy efficient robot (SpaceClimber 1) for extraterrestrial surface exploration, particularly for mobility in lunar craters. Estremera et al. [5] elaborated the development of crab and turning gaits for a hexapod robot, SILO-6, on a natural terrain containing uneven ground and forbidden zones as well as an application to humanitarian demining. Moro et al. [6] proposed an approach to directly map a range of gaits of a horse to a quadruped robot with an intention of generating a more life-like locomotion cycle. This work also presented the use of kinematic motion primitives in generating valid and stable walking, trotting, and galloping gaits that were tested on a compliant quadruped robot. In these works, the robots developed were generally effective in mimicking the gait cycles of their biological counterparts, but they suffered from high payload-to-machine-load ratio and high energy consumption.

Several approaches were studied in developing energy efficient walking machines. Reference [7] presented a set of rules towards improving energy efficiency in statically stable walking robots by comparing two-legged, namely, mammal and insect, configurations on a hexapod robotic platform. Reference [8] applied minimization criteria for optimizing energy consumption in a hexapod robot over every half a locomotion cycle, especially while walking on uneven terrains. Reference [9] put forward two different approaches to determine optimal feet forces and joint torques for six-legged robots towards minimizing energy consumption. Even though these works focused on the energy optimization problem, the robots experimented therein involved a series of links with multiple actuators to realize walking motion. Jansen [10], a Dutch kinetic artist, proposed an unconventional closed-kinematic-chain-based approach which requires actuation at only a single per leg through mapping internal cyclic motion into elliptical ones. Various aspects of the Jansen mechanism have been studied 


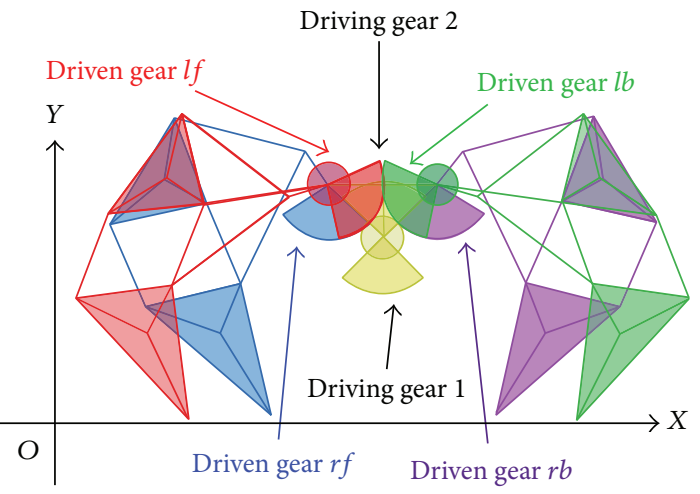

(a) $2 \mathrm{D}$

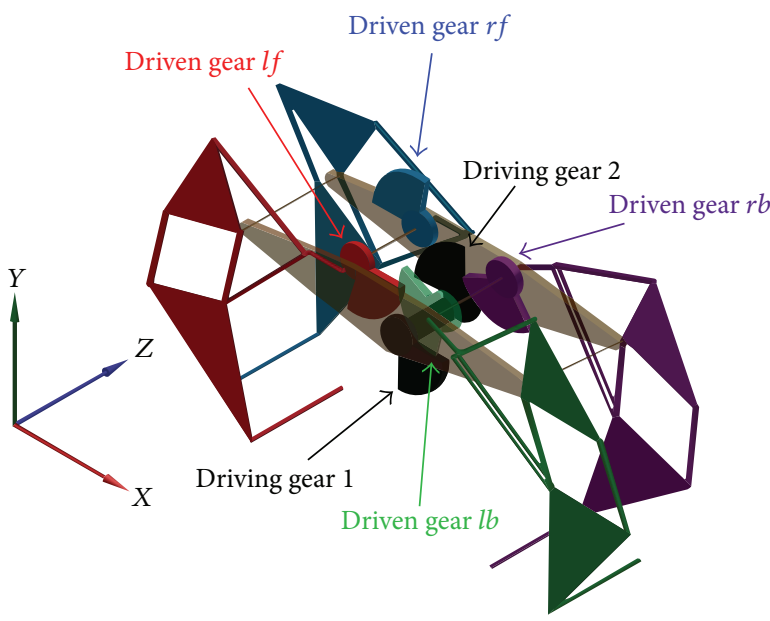

(b) $3 \mathrm{D}$

FIGURE 1: Schematic diagram of the quadruped robot composed of four Jansen linkage mechanisms and five gears which are driven by only one input attached to the bright yellow gear. The red, sky blue, green, and purple represent left-fore, right-fore, left-back, and right-back, respectively.

by a number of researchers. Reference [11] proposed an extension of the Theo Jansen mechanism by introducing an additional up-down motion in the linkage center for realizing new gait cycles with about ten times the height of the original for climbing over obstacles. Vector loop and simple geometric methods were used in conjunction with software tools such as ProEngineer and SAM for analyzing forward kinematics of the Theo Jansen mechanism in [12]. An attempt to optimize the leg geometry of the Theo Jansen mechanism using genetic algorithm was presented in [13]. The work explored the stability limits and tractive abilities while validating the kinematic and kinetic models through experiments with hardware prototypes. However, these two works did not provide dynamics analysis and discussions. Reference [14] conducted a preliminary dynamic analysis using the superposition method with the intention of optimizing the Theo Jansen mechanism; but this work is incomplete, without details on the analysis and discussions of equivalent Lagrange's equation. In fact, the complete dynamic analysis involving constraint forces and equivalent Lagrange's equation of motion is necessary for any meaningful extension and/or optimization of legged systems based on Theo Jansen mechanisms.

Lagrange's method [15-17] is famous for deriving dynamic models. Nevertheless, sometimes it is very difficult to build the dynamic model of a linkage mechanism, such as the Theo Jansen linkage, by using this method because the forward kinematics of the system becomes cumbersome. In this paper, the projection method [18-20] is applied to derive the complete dynamic model of a four-legged robot driven by a single actuator and composed of Theo Jansen mechanisms that was originally proposed by Studer [21]. In comparison with the conventional approaches, Lagrange's, Gibbs-Appel, and Kane's, for example, the projection method has been observed to be more intuitive in nature and compact $[18,19,22]$. By using this approach, the linkage mechanism can be assumed as a simple holonomic system and the model is derived by focusing on its constraints [22]. The dynamic model of the whole system can be established by separating the system into a few subcomponents and then integrating their dynamic models [23].

The complete dynamic analysis of Theo Jansen mechanisms is essential and will be conducted in the following contents. For the sake of convenience, the Jansen linkage or Jansen mechanism will be referred to as Theo Jansen mechanisms hereafter. The rest of this paper is organized as follows: In Section 2, the free-fall dynamic modeling of the one-actuator four-legged robot with Theo Jansen mechanisms is presented; this consists of the models of each Jansen leg and the corresponding gear system. In Section 3, the reaction force from the ground and friction are taken into account to build the plant dynamic model. The control system is designed based on energy control in Section 4. Numerical simulations are presented in Section 5. Finally, concluding remarks are given in Section 6.

\section{Free-Fall Model}

The schematic diagram of the quadruped robot is shown in Figure 1. This quadruped robot is composed of four Jansen linkage mechanisms and five gears which are driven by only one input attached to the bright yellow gear. The colors red, blue, green, and purple represent legs of left-fore, right-fore, left-back, and right-back, respectively. As a matter of practical convenience, we define $i=r, l$ and $j=f, b$ in this paper unless otherwise noted where $r, l, f$, and $b$ represent the right leg, left leg, fore leg, and back leg, respectively. The robot contacts with the ground on only toes, and the $x$-axis represents the ground. Because the four legs are the same Jansen linkage mechanisms, we can have the free-fall model of the whole robot by establishing the dynamic models of the 


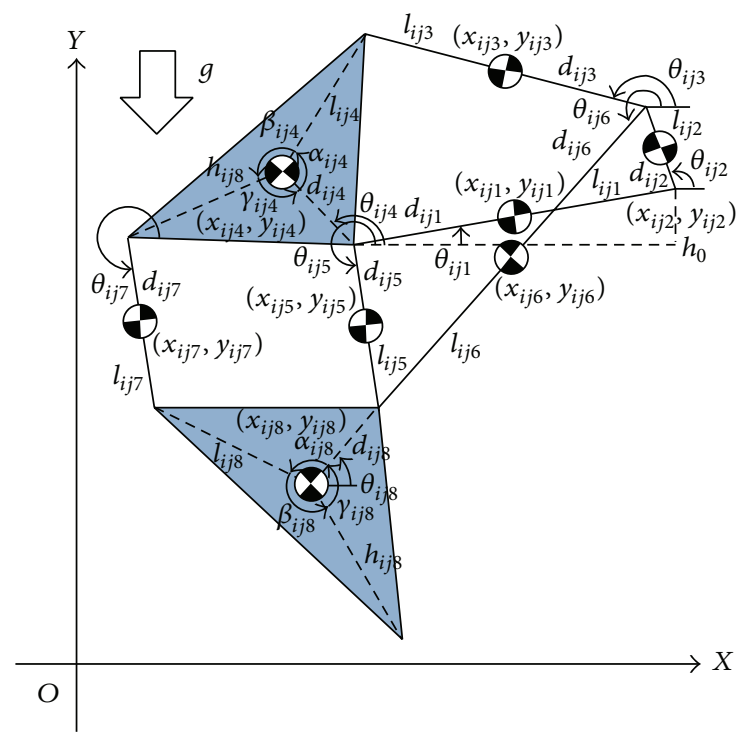

FIGURE 2: Schematic diagram of the Theo Jansen linkage mechanism with all parameters defined. The solid lines $l_{i j 1}, l_{i j 2}, l_{i j 3}, l_{i j 5}, l_{i j 6}, l_{i j 7}$ are the links; the two blue triangular parts are treated as the mass components; the dashed lines connect the gravity center of the mass and the corners of the triangle parts.

TABLE 1: Physical parameters of the Theo Jansen linkage mechanism $(\zeta, \xi=1, \ldots, 8)$.

\begin{tabular}{lcc}
\hline Parameter & Notation & Value \\
\hline Inertia moment $\left[\mathrm{kg} \cdot \mathrm{m}^{2}\right]$ & $J_{i j \zeta}$ & 0.01 \\
\hline Mass $[\mathrm{kg}]$ & $m_{i j \zeta}$ & 0.1 \\
\hline Viscous friction coefficient $[\mathrm{Nm} \cdot \mathrm{s} / \mathrm{rad}]$ & $C_{i j \zeta \xi}$ & 0.01 \\
\hline Height of link-1 $[\mathrm{m}]$ & $h_{0}$ & 0.02316 \\
\hline Length of link $[\mathrm{m}]$ & $L_{1, \ldots, 12}$ & \\
\hline Length from extremity to gravity center $[\mathrm{m}]$ & $d_{i j \zeta}$ & \\
\hline Length from gravity center to end $[\mathrm{m}]$ & $l_{i j \zeta}$ & \\
\hline & $d_{i j\{4,8\}}$ & \\
Length from gravity center to corner of triangle & $l_{i j\{4,8\}}$ & \\
[m] & $h_{i j\{4,8\}}$ & \\
& $\alpha_{i j\{4,8\}}$ & \\
& $\beta_{i j\{4,8\}}$ & \\
Central angle of triangle $[\mathrm{m}]$ & $\gamma_{i j\{4,8\}}$ & \\
&
\end{tabular}

single Jansen linkage and the gear system independently and then integrating the two models.

2.1. Dynamic Model of Jansen Linkage Mechanism. The schematic diagram and the coordinate system of a single Jansen linkage mechanism are illustrated in Figure 2. The physical parameters of the Jansen linkage mechanisms are tabulated in Table 1. The lengths of links adopted for the design in this study are listed in Table 3 [24]. It is assumed that the gravity center of every link locates on the center of the specified link, and the gravity center of each triangle locates
TABLE 2: Kinematic parameters of the Theo Jansen linkage mechanism.

\begin{tabular}{lc}
\hline Parameter & Value \\
\hline$d_{i j 4}$ & $0.0606657 \mathrm{~m}$ \\
$l_{i j 4}$ & $0.0844427 \mathrm{~m}$ \\
$h_{i j 4}$ & $0.0882124 \mathrm{~m}$ \\
$d_{i j 8}$ & $0.0739384 \mathrm{~m}$ \\
$l_{i j 8}$ & $0.102658 \mathrm{~m}$ \\
$h_{i j 8}$ & $0.126316 \mathrm{~m}$ \\
$\alpha_{i j 4}$ & $1.87097 \mathrm{rad}$ \\
$\beta_{i j 4}$ & $2.4248 \mathrm{rad}$ \\
$\gamma_{i j 4}$ & $1.98742 \mathrm{rad}$ \\
$\alpha_{i j 8}$ & $1.57408 \mathrm{rad}$ \\
$\beta_{i j 8}$ & $2.51629 \mathrm{rad}$ \\
$\gamma_{i j 8}$ & $2.19281 \mathrm{rad}$ \\
\hline
\end{tabular}

TABLE 3: The lengths of links adopted for the Theo Jansen linkage mechanism of this study.

\begin{tabular}{lc}
\hline Link & Value $(\mathrm{m})$ \\
\hline$L_{1}$ & 0.16218 \\
$L_{2}$ & 0.05417 \\
$L_{3}$ & 0.174425 \\
$L_{4}$ & 0.117645 \\
$L_{5}$ & 0.12567 \\
$L_{6}$ & 0.11754 \\
$L_{7}$ & 0.12671 \\
$L_{8}$ & 0.17974 \\
$L_{9}$ & 0.19161 \\
$L_{10}$ & 0.16169 \\
$L_{11}$ & 0.11967 \\
$L_{12}$ & 0.217995 \\
\hline
\end{tabular}

on vertex of three median lines. The angle between the gravity center and the corners of the triangle and the length from the gravity center to the corners of the triangle are calculated utilizing the law of cosines and are given in Table 2.

To construct the constraint-free dynamic model based on Figure 2, first of all, the generalized coordinate of this system is defined as

$$
\mathbf{x}_{p i j}=\left[\begin{array}{lllllll}
\theta_{i j 1} & x_{i j 1} & y_{i j 1} & \cdots & \theta_{i j 8} & x_{i j 8} & y_{i j 8}
\end{array}\right]^{T},
$$

where $\theta_{i j k}(k=1, \ldots, 8)$ represents the absolute angle of each link, and $\left(x_{i j k}, y_{i j k}\right)(k=1, \ldots, 8)$ represents the absolute coordinate of each link. Since the constraint-free model means a model in which every element has the highest degree of freedom as shown in Figure 3, it is only necessary to formulate motion equations around every gravity center according to Newton's second law to derive the constraintfree model. In a linkage mechanism like the system of this paper, the gravity and viscous friction forces are generated to each link $[25,26]$. Because the viscous friction forces are generated in proportion to velocities, the matrix form motion equation around the gravity center (i.e., the constraint-free 
model) is represented as (2) with attention that the viscous friction forces are generated by corresponding to the relative velocity

$$
\mathbf{M}_{i j} \ddot{\mathbf{x}}_{p i j}=\mathbf{h}_{i j}
$$

where the generalized mass matrix $\mathbf{M}_{i j}$ and the generalized force matrix $\mathbf{h}_{i j}$ are defined as

$$
\begin{aligned}
& \mathbf{M}_{i j}=\operatorname{diag}\left(J_{i j 1}, m_{i j 1}, m_{i j 1}, \ldots, J_{i j 8}, m_{i j 8}, m_{i j 8}\right), \\
& \mathbf{h}_{i j}
\end{aligned}
$$$$
\left[\begin{array}{c}
C_{i j 12} \dot{\phi}_{i j 12}+C_{i j 14} \dot{\phi}_{i j 14} \\
0 \\
-m_{i j 1} g \\
-C_{i j 12} \dot{\phi}_{i j 12}+C_{i j 23} \dot{\phi}_{i j 23} \\
0 \\
-m_{i j 2} g \\
-C_{i j 23} \dot{\phi}_{i j 23}+C_{i j 34} \dot{\phi}_{i j 34}+C_{i j 36} \dot{\phi}_{i j 36} \\
0 \\
-m_{i j 3} g \\
-C_{i j 14} \dot{\phi}_{i j 14}-C_{i j 34} \dot{\phi}_{i j 34}+C_{i j 45} \dot{\phi}_{i j 45}+C_{i j 47} \dot{\phi}_{i j 47} \\
0 \\
-m_{i j 4} g \\
-C_{i j 45} \dot{\phi}_{i j 45}+C_{i j 56} \dot{\phi}_{i j 56}+C_{i j 58} \dot{\phi}_{i j 58} \\
0 \\
-m_{i j 5} g \\
-C_{i j 68} \dot{\phi}_{i j 68}-C_{i j 78} \dot{\phi}_{i j 78} \\
0 \\
-m_{i j 47} \dot{\phi}_{i j 47}+C_{i j 78} \dot{\phi}_{i j 78} \\
0 \\
-C_{i j 36} \dot{\phi}_{i j 36}-C_{i j 56} \dot{\phi}_{i j 56}+C_{i j 68} \dot{\phi}_{i j 68} \\
0 \\
-m_{i j 6} g
\end{array}\right]
$$

where $\dot{\phi}_{i j \zeta \xi}=\dot{\theta}_{i j \xi}-\dot{\theta}_{i j \zeta},(\zeta, \xi=1, \ldots, 8)$.

In the next step, the constraint matrix $\mathbf{C}_{i j}$ which holds $\mathbf{C}_{i j} \dot{\mathbf{x}}_{p i j}=0$ is formulated from constraint conditions. The coordinates of the gravity center and the length of each link satisfy a certain relationship geometrically. For example, $x_{i j 2}$, $y_{i j 2}$ can be represented as follows:

$$
\begin{aligned}
& x_{i j 2}=x_{i j 1}+l_{i j 1} \cos \theta_{i j 1}+d_{i j 2} \cos \theta_{i j 2}, \\
& y_{i j 2}=y_{i j 1}+l_{i j 1} \sin \theta_{i j 1}+d_{i j 2} \sin \theta_{i j 2}
\end{aligned}
$$

By moving right side to the other side, (4) is represented as follows:

$$
\begin{gathered}
x_{i j 2}-x_{i j 1}-l_{i j 1} \cos \theta_{i j 1}-d_{i j 2} \cos \theta_{i j 2}=0, \\
y_{i j 2}-y_{i j 1}-l_{i j 1} \sin \theta_{i j 1}-d_{i j 2} \sin \theta_{i j 2}=0 .
\end{gathered}
$$

In addition, all other gravity centers can be represented as well as above. And, by forming these as matrix, the holonomic constraint relevant to the gravity center is formulated as follows:

$$
\left.\boldsymbol{\Phi}_{h i j} \begin{array}{c}
x_{i j 2}-x_{i j 1}-l_{i j 1} \cos \theta_{i j 1}-d_{i j 2} \cos \theta_{i j 2} \\
y_{i j 2}-y_{i j 1}-l_{i j 1} \sin \theta_{i j 1}-d_{i j 2} \sin \theta_{i j 2} \\
x_{i j 3}-x_{i j 2}-l_{i j 2} \cos \theta_{i j 2}-d_{i j 3} \cos \theta_{i j 3} \\
y_{i j 3}-y_{i j 2}-l_{i j 2} \sin \theta_{i j 2}-d_{i j 3} \sin \theta_{i j 3} \\
x_{i j 4}-x_{i j 3}-l_{i j 3} \cos \theta_{i j 3}-l_{i j 4} \cos \left(\theta_{i j 4}+\alpha_{i j 4}\right) \\
y_{i j 4}-y_{i j 3}-l_{i j 3} \sin \theta_{i j 3}-l_{i j 4} \sin \left(\theta_{i j 4}+\alpha_{i j 4}\right) \\
x_{i j 4}-x_{i j 1}+d_{i j 1} \cos \theta_{i j 1}-d_{i j 4} \cos \theta_{i j 4} \\
y_{i j 4}-y_{i j 1}+d_{i j 1} \sin \theta_{i j 1}-d_{i j 4} \sin \theta_{i j 4} \\
x_{i j 5}-x_{i j 1}+d_{i j 1} \cos \theta_{i j 1}-d_{i j 5} \cos \theta_{i j 5} \\
y_{i j 5}-y_{i j 1}+d_{i j 1} \sin \theta_{i j 1}-d_{i j 5} \sin \theta_{i j 5} \\
x_{i j 6}-x_{i j 5}-l_{i j 5} \cos \theta_{i j 5}+l_{i j 6} \cos \theta_{i j 6} \\
y_{i j 6}-y_{i j 5}-l_{i j 5} \sin \theta_{i j 5}+l_{i j 6} \sin \theta_{i j 6} \\
x_{i j 6}-x_{i j 2}-l_{i j 2} \cos \theta_{i j 2}-d_{i j 6} \cos \theta_{i j 6} \\
y_{i j 6}-y_{i j 2}-l_{i j 2} \sin \theta_{i j 2}-d_{i j 6} \sin \theta_{i j 6} \\
x_{i j 7}-x_{i j 4}+h_{i j 4} \cos \left(\theta_{i j 4}-\gamma_{i j 4}\right)-d_{i j 7} \cos \theta_{i j 7} \\
y_{i j 7}-y_{i j 4}+h_{i j 4} \sin \left(\theta_{i j 4}-\gamma_{i j 4}\right)-d_{i j 7} \sin \theta_{i j 7} \\
x_{i j 8}-x_{i j 5}-l_{i j 5} \cos \theta_{i j 5}+d_{i j 8} \cos \theta_{i j 8} \\
y_{i j 8}-y_{i j 5}-l_{i j 5} \sin \theta_{i j 5}+d_{i j 8} \sin \theta_{i j 8} \\
x_{i j 8}-x_{i j 7}-l_{i j 7} \cos \theta_{i j 7}+l_{i j 8} \cos \left(\theta_{i j 8}+\alpha_{i j 8}\right) \\
y_{i j 8}-y_{i j 7}-l_{i j 7} \sin \theta_{i j 7}+l_{i j 8} \sin \left(\theta_{i j 8}+\alpha_{i j 8}\right)
\end{array}\right]
$$

Then the constraint matrix is defined as

$$
\mathrm{C}_{i j}:=\frac{\partial \Phi_{h i j}}{\partial \mathbf{x}_{p i j}} .
$$

The constraint dynamical system can thus be formulated by adding the constraint term (7) with Lagrange's multipliers $\boldsymbol{\lambda}_{i j}$ to (2) as

$$
\mathbf{M}_{i j} \ddot{\mathbf{x}}_{p i j}=\mathbf{h}_{i j}+\mathbf{C}_{i j}^{T} \lambda_{i j}
$$

Moreover, the degree of freedom of the unconstraint system is found to be 24 from (1). The degrees of freedom should be constrained by 20 holonomic constraints in this 


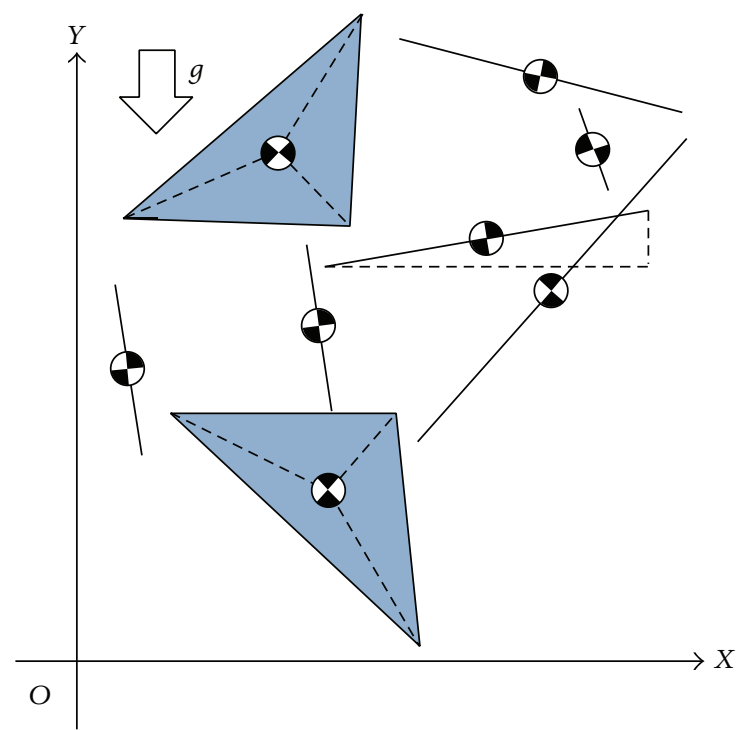

Figure 3: Schematic diagram of the constraint-free model. In this system, all gravity centers have the highest degree of freedom.

system. Therefore, the degree of freedom of the constrained dynamical system (8) is 4 .

The tangent speed of the constrained system is denoted as

$$
\dot{\mathbf{q}}_{i j}=\left[\begin{array}{llll}
\dot{\theta}_{i j 1} & \dot{x}_{i j 1} & \dot{y}_{i j 1} & \dot{\theta}_{i j 2}
\end{array}\right]^{T} .
$$

Setting a partition symbolically as $\mathbf{v}=\left[\begin{array}{ll}\dot{\mathbf{q}}_{i j}^{T} & \mathbf{v}_{\mathbf{D} i j}^{T}\end{array}\right]^{T}$ where $\mathbf{v}_{\mathbf{D} i j}$ shows dependent velocities with respect to $\dot{\mathbf{q}}_{i j}, \mathbf{C}_{i j}$ is decomposed into $\mathbf{C}_{i j}=\left[\begin{array}{ll}\mathbf{C}_{i j 1} & \mathbf{C}_{i j 2}\end{array}\right]$ satisfying $\mathbf{C}_{i j} \dot{\mathbf{x}}_{p i j}=$ $\mathbf{C}_{i j 1} \dot{\mathbf{q}}_{i j}+\mathbf{C}_{i j 2} \mathbf{v}_{\mathbf{D} i j} . \mathbf{D}_{i j}$ is the orthogonal complement matrix to $\mathbf{C}_{i j}$ satisfying $\mathbf{C}_{i j} \mathbf{D}_{i j}=0, \dot{\mathbf{x}}_{p i j}=\mathbf{D}_{i j} \dot{\mathbf{q}}_{i j}$, and

$$
\mathbf{D}_{i j}=\left[\begin{array}{c}
\mathbf{I}^{4 \times 4} \\
-\mathbf{C}_{i j 2}^{-1} \mathbf{C}_{i j 1}
\end{array}\right],
$$

where $\mathbf{I}^{4 \times 4}$ represents the identity matrix $\mathbf{I} \in \mathbf{R}^{4 \times 4}$.

Finally, multiplying (8) by $\mathbf{D}_{i j}^{T}$ from the left side and substituting the coordinate transformation $\dot{\mathbf{x}}_{p i j}=\mathbf{D}_{i j} \dot{\mathbf{q}}_{i j}$ into (8) can eliminate the constraint term with $\boldsymbol{\lambda}_{i j}$, and the dynamic model of the Jansen linkage mechanism is

$$
\mathbf{D}_{i j}^{T} \mathbf{M}_{i j} \mathbf{D}_{i j} \ddot{\mathbf{q}}_{i j}+\mathbf{D}_{i j}^{T} \mathbf{M}_{i j} \dot{\mathbf{D}}_{i j} \dot{\mathbf{q}}_{i j}=\mathbf{D}_{i j}^{T} \mathbf{h}_{i j} .
$$

2.2. Dynamic Model of Gear System. After the establishment of the dynamic model of the Jansen linkage mechanism, the dynamic model of the gear system [13] is derived following the same steps as Section 2.1. Table 4 gives the notations and values of the physical parameters of the gear system. Figure 4 illustrates the schematic diagram and coordinate system of the gear system consisting of six gears where the subscripts $\iota$ and $m$ represent the frame of the gears and the driving gears including, respectively. The two driving gears are driven by a single actuator and rotate synchronously. Meanwhile, the four driven gears are driven by the two driving gears. The
TABLE 4: Physical parameters of the gear system.

\begin{tabular}{lcc}
\hline Parameter & Notation & Value \\
\hline \multirow{2}{*}{ Inertia moment $\left[\mathrm{kg} \cdot \mathrm{m}^{2}\right]$} & $J_{\iota}$ & 0.01 \\
& $J_{m}$ & 0.01 \\
& $J_{i j}$ & 0.01 \\
\hline \multirow{2}{*}{ Mass [kg] } & $m_{\iota}$ & 1.0 \\
& $m_{m}$ & 0.1 \\
& $m_{i j}$ & 0.1 \\
\hline \multirow{2}{*}{ Viscous friction coefficient $[\mathrm{Nm} \cdot \mathrm{s} / \mathrm{rad}]$} & $C_{\iota}$ & 0.01 \\
& $C_{m}$ & 0.01 \\
Center distance of gears [m] & $C_{i j}$ & 0.01 \\
\hline \multirow{2}{*}{ Length of frame $[\mathrm{m}]$} & $d$ & 0.1 \\
\hline \multirow{2}{*}{ Radius of gears $[\mathrm{m}]$} & $l_{m}$ & $d / \sqrt{2}$ \\
& $l_{i j}$ & $d / \sqrt{2}$ \\
\hline
\end{tabular}

frame is used to fix all the gears. All gears are noncircular gears [27-29] which vary their gear ratios during rotation, but the driven gears rotate one revolution with respect to one revolution of the driving gear. In addition, the gear ratio of each gear varies depending on the angle between the driving gear and the gear frame. Based on these conditions, the gear ratio is defined as

$$
\begin{aligned}
& r_{m i j} \\
& = \begin{cases}d \frac{\theta_{B}-\theta_{A}}{\theta_{B}-\theta_{A}+\pi / 2}, & \text { (High Speed Ratio), } \\
d \frac{\theta_{A}+2 \pi-\theta_{B}}{\theta_{A}+2 \pi-\theta_{B}+3 \pi / 2}, & \text { (Low Speed Ratio), }\end{cases} \\
& r_{i j} \\
& = \begin{cases}d \frac{\pi / 2}{\theta_{B}-\theta_{A}+\pi / 2}, & \text { (High Speed Ratio), } \\
d \frac{3 \pi / 2}{\theta_{A}+2 \pi-\theta_{B}+3 \pi / 2}, & \text { (Low Speed Ratio), }\end{cases}
\end{aligned}
$$

where $\theta_{A}$ and $\theta_{B}$ are with respect to each gait pattern of the Jansen linkage mechanism $\left(\theta_{A}=1.05 \mathrm{rad}\right.$ and $\theta_{B}=5.55 \mathrm{rad}$ in this paper).

The constraint-free model is derived according to Figure 4 with parameters defined in Table 4 . The generalized coordinate of this system is given:

$$
\mathbf{x}_{p g}=\left[\begin{array}{lllllllll}
\theta_{\iota} & x_{\iota} & y_{\iota} & \theta_{m} & x_{m} & y_{m} & \theta_{i j} & x_{i j} & y_{i j}
\end{array}\right]^{T},
$$

where $\theta_{\iota, m, i j}$ and $\left(x_{\iota, m, i j}, y_{\iota, m, i j}\right)$ represent the absolute angles and coordinates of the gear frame, the driving and driven gears, respectively. With the generalized coordinate (13), equations of motion concerned with the gear system are formulated below:

$$
\mathbf{M}_{g} \ddot{\mathbf{x}}_{p g}=\mathbf{h}_{g},
$$




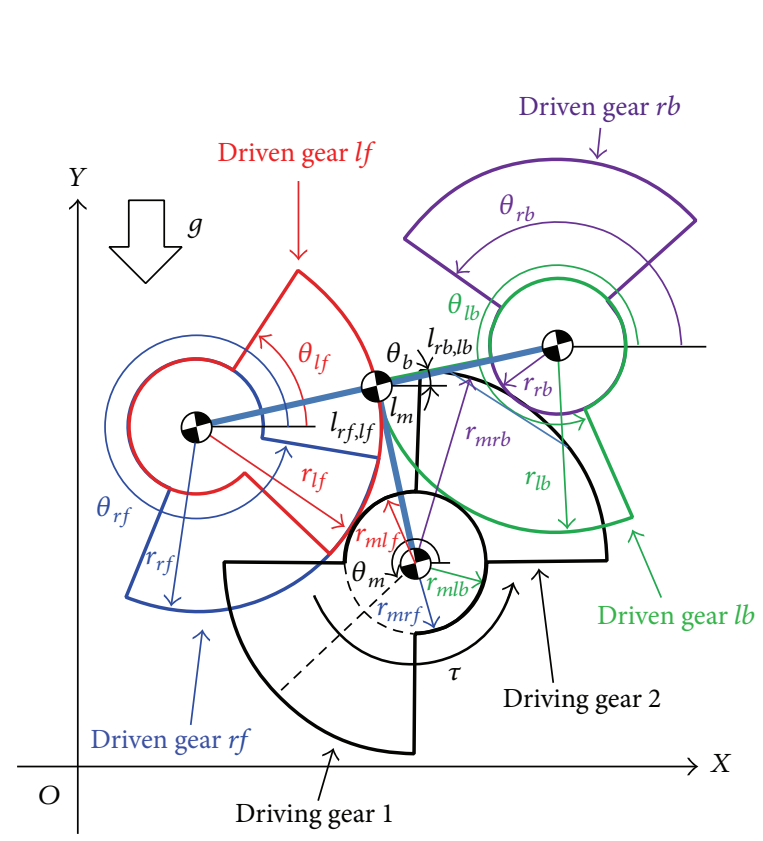

(a) $2 \mathrm{D}$

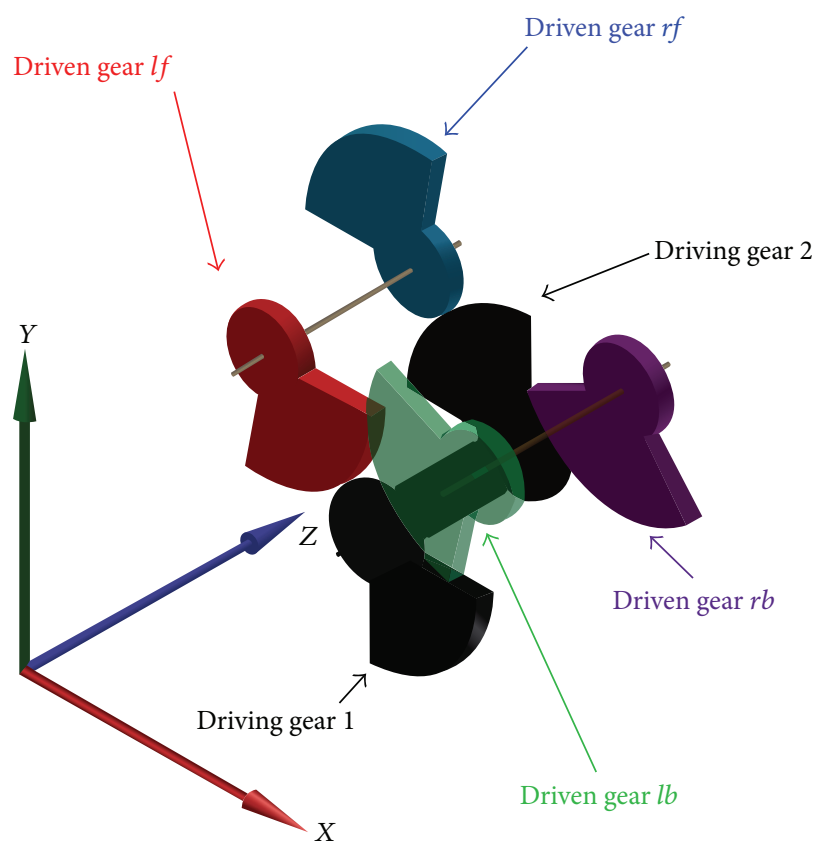

(b) $3 \mathrm{D}$

FIGURE 4: Schematic diagram of the gear system with parameters defined. The black-margined gear represents the driving gear, and the other gears represent the driven gears. The driving gear 1 and the driving gear 2 rotate synchronously.

where

$$
\begin{gathered}
\mathbf{M}_{g}=\operatorname{diag}\left(J_{l}, m_{l}, m_{l}, J_{m}, m_{m}, m_{m}, J_{i j}, m_{i j}, m_{i j}\right), \\
\mathbf{h}_{g}=\left[\begin{array}{c}
-\tau+C_{m}\left(\dot{\theta}_{\iota}-\dot{\theta}_{m}\right)+C_{i j}\left(\dot{\theta}_{\iota}-\dot{\theta}_{i j}\right) \\
0 \\
-m_{\iota} g \\
\tau-C_{m}\left(\dot{\theta}_{\iota}-\dot{\theta}_{m}\right) \\
0 \\
-m_{m} g \\
-C_{r f}\left(\dot{\theta}_{\iota}-\dot{\theta}_{r f}\right) \\
0 \\
-m_{r f} \mathcal{g} \\
-C_{r b}\left(\dot{\theta}_{\iota}-\dot{\theta}_{r b}\right) \\
0 \\
-m_{r b} g \\
-C_{l f}\left(\dot{\theta}_{\iota}-\dot{\theta}_{l f}\right) \\
0 \\
-m_{l f} g \\
-C_{l b}\left(\dot{\theta}_{\iota}-\dot{\theta}_{l b}\right) \\
0 \\
-m_{l b} g
\end{array}\right],
\end{gathered}
$$

with $\tau$ representing the input torque.
The coordinates of the gravity center and the length of each link are correlated geometrically. And the rotation angles and radius of gears also conform to some relationship. Based on these relationships, the holonomic constraints are formulated as follows: For the gravity centers and gear ratio, the holonomic constraints are formulated as follows:

$$
\Phi_{h g}=\left[\begin{array}{c}
x_{m}-x_{\iota}-l_{m} \sin \theta_{\iota} \\
y_{m}-y_{\iota}-l_{m} \cos \theta_{\iota} \\
\theta_{r f}+\frac{r_{m r f}}{r_{r f}} \theta_{m}-\frac{r_{m r f}+r_{r f}}{r_{r f}} \theta_{\iota} \\
x_{r f}-x_{\iota}+l_{r f} \cos \theta_{\iota} \\
y_{r f}-y_{\iota}+l_{r f} \sin \theta_{\iota} \\
\theta_{r b}+\frac{r_{m r b}}{r_{r b}} \theta_{m}-\frac{r_{m r b}+r_{r b}}{r_{r b}} \theta_{\iota} \\
x_{r b}-x_{\iota}-l_{r b} \cos \theta_{\iota} \\
y_{r b}-y_{\iota}-l_{r b} \sin \theta_{\iota} \\
\theta_{l f}+\frac{r_{m l f}}{r_{l f}} \theta_{m}-\frac{r_{m l f}+r_{l f}}{r_{l f}} \theta_{\iota} \\
x_{l f}-x_{\iota}+l_{l f} \cos \theta_{\iota} \\
y_{l f}-y_{\iota}+l_{l f} \sin \theta_{\iota} \\
\theta_{m l b}+\frac{r_{m l b}+r_{l b}}{r_{l b}} \theta_{\iota} \\
x_{l b}-x_{\iota}-l_{l b} \cos \theta_{\iota} \\
y_{l b}-y_{\iota}-l_{l b} \sin \theta_{\iota}
\end{array}\right]=0 .
$$


The corresponding constraint matrix $\mathbf{C}_{g}$ which holds $\mathbf{C}_{g} \dot{\mathbf{x}}_{p g}=0$ is defined as

$$
\mathbf{C}_{g}:=\frac{\partial \Phi_{h g}}{\partial \mathbf{x}_{p g}} .
$$

Subsequently, the constraint dynamical model can thus be obtained by adding the constraint matrix with Lagrange's multipliers $\lambda_{g}$ to (14) as

$$
\mathbf{M}_{g} \ddot{\mathbf{x}}_{p g}=\mathbf{h}_{g}+\mathbf{C}_{g}^{T} \boldsymbol{\lambda}_{g} .
$$

The degrees of freedom of the unconstraint system are found to be 18 from (13) which should be constrained by 14 holonomic constraints in this system. Therefore, the degree of freedom of the constrained dynamical system is 4 . The tangent speed of the constrained system is

$$
\dot{\mathbf{q}}_{g}=\left[\begin{array}{llll}
\dot{\theta}_{l} & \dot{x}_{l} & \dot{y}_{l} & \dot{\theta}_{m}
\end{array}\right]^{T} \text {. }
$$

The orthogonal complement matrix $\mathbf{D}_{g}$ is obtained according to the same steps as Section 2.1. Multiplying (18) by $\mathbf{D}_{g}^{T}$ from the left side and substituting the coordinate transformation $\dot{\mathbf{x}}_{p g}=\mathbf{D}_{g} \dot{\mathbf{q}}_{g}$ into (18) yield the dynamic model of Figure 4 :

$$
\mathbf{D}_{g}^{T} \mathbf{M}_{g} \mathbf{D}_{g} \ddot{\mathbf{q}}_{g}+\mathbf{D}_{g}^{T} \mathbf{M}_{g} \dot{\mathbf{D}}_{g} \dot{\mathbf{q}}_{g}=\mathbf{D}_{g}^{T} \mathbf{h}_{g} .
$$

2.3. System Integration. Up till now the dynamic models of the Jansen linkage mechanisms and the gear system have already been established. Then the whole constraint-free dynamic model of the quadruped robot can be obtained by integrating (11) and (20):

$$
\mathbf{M} \ddot{\mathbf{x}}_{p}=\mathbf{h},
$$

where

$$
\begin{aligned}
& \mathbf{x}_{p}=\left[\begin{array}{lllll}
\mathbf{q}_{g}^{T} & \mathbf{q}_{r f}^{T} & \mathbf{q}_{l f}^{T} & \mathbf{q}_{r b}^{T} & \mathbf{q}_{l b}^{T}
\end{array}\right]^{T}, \\
& \mathbf{M}=\operatorname{diag}\left(\mathbf{D}_{r f}^{T} \mathbf{M}_{r f} \mathbf{D}_{r f}, \mathbf{D}_{l f}^{T} \mathbf{M}_{l f} \mathbf{D}_{l f}, \mathbf{D}_{r b}^{T} \mathbf{M}_{r b} \mathbf{D}_{r b},\right. \\
& \left.\mathbf{D}_{l b}^{T} \mathbf{M}_{l b} \mathbf{D}_{l b}\right) \text {, } \\
& \mathbf{h}=\left[\begin{array}{c}
\mathbf{D}_{r f}^{T} \mathbf{h}_{r f}-\mathbf{D}_{r f}^{T} \mathbf{M}_{r f} \dot{\mathbf{D}}_{r f} \dot{\mathbf{q}}_{r f} \\
\mathbf{D}_{l f}^{T} \mathbf{h}_{l f}-\mathbf{D}_{l f}^{T} \mathbf{M}_{l f} \dot{\mathbf{D}}_{l f} \dot{\mathbf{q}}_{l f} \\
\mathbf{D}_{r b}^{T} \mathbf{h}_{r b}-\mathbf{D}_{r b}^{T} \mathbf{M}_{r b} \dot{\mathbf{D}}_{r b} \dot{\mathbf{q}}_{r b} \\
\mathbf{D}_{l b}^{T} \mathbf{h}_{l b}-\mathbf{D}_{l b}^{T} \mathbf{M}_{l b} \dot{\mathbf{D}}_{l b} \dot{\mathbf{q}}_{l b}
\end{array}\right] .
\end{aligned}
$$

Again, given the holonomic constraints of the gravity center of each link,

$$
\Phi_{h}=\left[\begin{array}{c}
\theta_{r f 1}-\theta_{\iota}-\tan ^{-1}\left(\frac{L_{1}}{h_{0}}\right) \\
x_{r f 1}-x_{\iota}+l_{r f} \cos \theta_{\iota}+l_{r f 1} \cos \theta_{r f 1} \\
y_{r f 1}-y_{\iota}+l_{r f} \sin \theta_{\iota}+l_{r f 1} \sin \theta_{r f 1} \\
\theta_{r f 2}+\frac{r_{m r f}}{r_{r f}} \theta_{m}-\frac{r_{m r f}+r_{r f}}{r_{r f}} \theta_{\iota} \\
\theta_{r b 1}-\theta_{\iota}-\tan ^{-1}\left(-\frac{L_{1}}{h_{0}}\right) \\
x_{r b 1}-x_{\iota}-l_{r b} \cos \theta_{\iota}+l_{r b 1} \cos \theta_{r b 1} \\
y_{r b 1}-y_{\iota}-l_{r b} \sin \theta_{\iota}+l_{r b 1} \sin \theta_{r b 1} \\
\theta_{r b 2}+\frac{r_{m r b}}{r_{r b}} \theta_{m}-\frac{r_{m r b}+r_{r b}}{r_{r b}} \theta_{\iota} \\
\theta_{l f 1}-\theta_{\iota}-\tan \left(\frac{L_{1}}{h_{0}}\right) \\
x_{l f 1}-x_{\iota}+l_{l f} \cos \theta_{\iota}+l_{l f 1} \cos \theta_{l f 1} \\
y_{l f 1}-y_{\iota}+l_{l f} \sin \theta_{\iota}+l_{l f 1} \sin \theta_{l f 1} \\
\theta_{l f 2}+\frac{r_{m l f}}{r_{l f}} \theta_{m}-\frac{r_{m l f}+r_{l f}}{r_{l f}} \theta_{\iota} \\
\theta_{l b 1}-\theta_{\iota}-\tan { }^{-1}\left(-\frac{L_{1}}{h_{0}}\right) \\
x_{l b 1}-x_{\iota}-l_{l b} \cos \theta_{\iota}+l_{l b 1} \cos \theta_{l b 1} \\
y_{l b 1}-y_{\iota}-l_{l b} \sin \theta_{\iota}+l_{l b 1} \sin \theta_{l b 1} \\
\theta_{l b 2}+\frac{r_{m l b}}{r_{l b}} \theta_{m}-\frac{r_{m l b}+r_{l b}}{r_{l b}} \theta_{\iota}
\end{array}\right]=0,
$$

the constraint dynamical system is thus

$$
\mathbf{M} \ddot{\mathbf{x}}_{p}=\mathbf{h}+\mathbf{C}^{T} \boldsymbol{\lambda}
$$

where

$$
\mathbf{C}:=\frac{\partial \Phi_{h}}{\partial \mathbf{x}_{p}} .
$$

From (21) we find that the degrees of freedom of the unconstraint system are 20 and constrained by 16 holonomic constraints. Hence, the degree of freedom of the constrained dynamical system is 4 .

Finally, by multiplying (24) by $\mathbf{D}^{T}$ (where $\mathbf{D}$ is the orthogonal complement matrix) from the left side and substituting the coordinate transformation $\dot{\mathbf{x}}_{p}=\mathbf{D} \dot{\mathbf{q}}$ into (24), the dynamic free-falling model is

$$
\mathbf{D}^{T} \mathbf{M D} \ddot{\mathbf{q}}+\mathbf{D}^{T} \mathbf{M} \dot{\mathbf{D}} \dot{\mathbf{q}}=\mathbf{D}^{T} \mathbf{h},
$$

where

$$
\dot{\mathbf{q}}=\left[\begin{array}{llll}
\dot{\theta}_{\iota} & \dot{x}_{\iota} & \dot{y}_{\iota} & \dot{\theta}_{m}
\end{array}\right]^{T}
$$

is the tangent speed of the constrained system which is the same as that of the gear system. 


\section{Plant Model}

The dynamic model (26) is the free-fall model which does not include reaction force from the ground. However, such force exists and can not be neglected in real situation. In this section, the dynamic model including the floor reaction force and the collision with the ground is established by expanding the free-fall model.

Our previous study has shown that the toes of the Jansen linkage mechanisms slip consistently while walking [30]. Hence, the further dynamic model considering friction is formulated in this section by assuming that the coefficient of friction is uniform.

3.1. Contact with the Ground. We consider the floor reaction force by expanding the constraint matrix using the projection method [31]. Contacting the toes with the ground can be regarded as constraining the toes on the ground (i.e., on the $x$ axis). Thus, the floor reaction force is expressed by including the constraint when $y$-coordinate of the toe is less than 0 and the constraint force of the toes is greater than 0 (i.e., the force is generated from the ground to the robot). Since this constraint can be described by the position constraint which is the holonomic constraint, the constraint matrix is defined as

$$
\begin{aligned}
& \mathbf{C}_{p}=\left[\begin{array}{llll}
\mathbf{C}_{r f}^{T} & \mathbf{C}_{l f}^{T} & \mathbf{C}_{r b}^{T} & \mathbf{C}_{l b}^{T}
\end{array}\right]^{T}, \\
& \mathbf{C}_{i j}=\frac{\partial t_{i j}}{\partial \mathbf{x}_{p}}, \quad\left(t_{i j} \leq 0 \cap \lambda_{f i j}>0\right),
\end{aligned}
$$

where $t_{i j}=y_{i j 8}+h_{i j 8} \sin \left(\theta_{i j 8}-\gamma_{i j 8}\right)$ and $\lambda_{f i j}$ represents the floor reaction force. Also, by utilizing the constraint matrix, (24) is expanded as

$$
\mathbf{M} \ddot{\mathbf{x}}_{p}=\mathbf{h}+\left[\begin{array}{c}
\mathbf{C} \\
\mathbf{C}_{p}
\end{array}\right]^{T}\left[\begin{array}{l}
\lambda \\
\lambda_{p}
\end{array}\right],
$$

which can be projected to the tangent speed space using the orthogonal complement matrix $\mathbf{D}$, and then the motion equations including the contact on the floor are formulated as

$$
\mathbf{D}^{T} \mathbf{M D} \ddot{\mathbf{q}}+\mathbf{D}^{T} \mathbf{M} \dot{\mathbf{D}} \dot{\mathbf{q}}=\mathbf{D}^{T} \mathbf{h}+\mathbf{D}^{T} \mathbf{C}_{p}^{T} \boldsymbol{\lambda}_{p} .
$$

Next, the friction force shown in Figure 5 will be included in the model. Some linear/nonlinear friction models have been already proposed in [32-35]. In this study, one of the simplest friction models which is composed of only kinetic and viscous frictions is applied because it has been assumed that the coefficient of friction is uniform. The kinetic friction force is generated when the toe has a speed and the viscous friction force is generated in proportion to the speed $[25,26]$ of the toe. Hence the friction model $\mathbf{f}$ is represented as

$$
\mathbf{f}=\left[\begin{array}{llll}
\mathbf{f}_{r f}^{T} \mathbf{D}_{r f} & \mathbf{f}_{r b}^{T} \mathbf{D}_{r b} & \mathbf{f}_{l f}^{T} \mathbf{D}_{l f} & \mathbf{f}_{l b}^{T} \mathbf{D}_{l b}
\end{array}\right]^{T},
$$

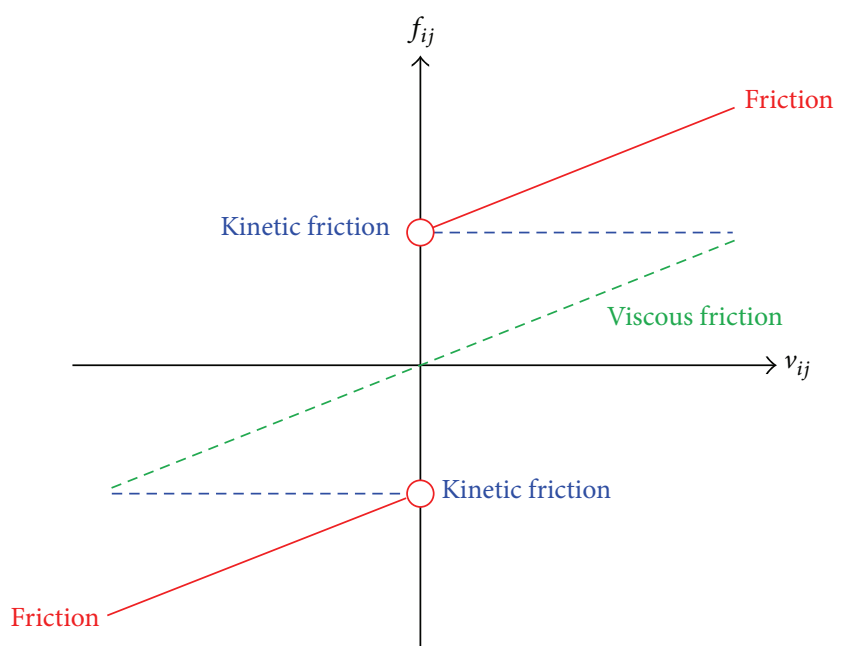

FIgURE 5: The diagram of the included friction model. $f_{i j}$ and $v_{i j}$ represent the friction force that generates the toe and the velocity of the toe, respectively. Blue line and green line represent the kinetic friction force and the viscous friction force, respectively. The composition of these two friction forces represents the actual generated friction force $f_{i j}$.

where

$$
\mathbf{f}_{i j}=\left[\begin{array}{c}
\mathbf{Z}^{21 \times 1} \\
h_{i j 8} \sin \left(\theta_{i j 8}-\gamma_{i j 8}\right)\left(\lambda_{i j} \mu_{d} \operatorname{sgn} \dot{\theta}_{i j 8}+\mu_{v} \dot{\theta}_{i j 8}\right) \\
\lambda_{i j} \mu_{d} \operatorname{sgn} \dot{x}_{i j 8}+\mu_{v} \dot{x}_{i j 8} \\
0
\end{array}\right],
$$

with kinetic friction coefficient $\mu_{d}$, viscous friction coefficient $\mu_{v}$, and zero matrix $\mathbf{Z}^{m \times n}$. Equation (31) is projected to the tangent speed space utilizing the orthogonal complement matrix and then substituted into (30). Finally, the motion equation of the quadruped robot with the Jansen linkage mechanisms considering the friction force is obtained:

$$
\mathbf{D}^{T} \mathbf{M D} \ddot{\mathbf{q}}+\mathbf{D}^{T} \mathbf{M D} \dot{\mathbf{q}}=\mathbf{D}^{T} \mathbf{h}+\mathbf{D}^{T} \mathbf{C}_{p}^{T} \boldsymbol{\lambda}_{p}+\mathbf{D}^{T} \mathbf{F}
$$

3.2. Collision with the Ground. As an assumption, the collision between the ground and the toes is a non-completely elastic collision. Based on the projection method, we assume that an impulse is input at the moment when the collision occurs, and the tangent speed after collision can be formulated from the one before collision $[31,36]$. The tangent speeds before and after collision are defined as $\dot{\mathbf{q}}^{-}$and $\dot{\mathbf{q}}^{+}$respectively, and their relationship can be expressed as

$$
\dot{\mathbf{q}}^{+}=\mathbf{H}\left(\mathbf{I}-\mathbf{M}^{-1} \mathbf{C}^{\prime T}\left(\mathbf{C}^{\prime} \mathbf{M}^{-1} \mathbf{C}^{\prime T}\right)^{-1} \mathbf{C}^{\prime}\right) \mathbf{D} \dot{\mathbf{q}}^{-}
$$

where

$$
\mathbf{H}=\left[\begin{array}{ll}
\mathbf{I}^{4 \times 4} & \mathbf{Z}^{4 \times 16}
\end{array}\right]
$$


and $\mathbf{C}^{\prime}$ represents the constraint matrix after collision which is defined as

$$
\mathbf{C}^{\prime}=\left[\begin{array}{c}
\mathbf{C} \\
\mathbf{C}_{p}
\end{array}\right] .
$$

\section{Control System Design}

So far we have the dynamic model taking into account the floor reaction and friction forces. However, the coefficient of friction is unknown in general as it is difficult to use the dynamic model into the controller. Therefore, the controller is designed by utilizing the approximate model based on the approximation conditions [37].

4.1. Approximate Model. Following two approximation conditions set based on characteristics of the model and mechanism, we could have the approximate model.

The first approximation condition is the contact with the ground by two legs consistently. The model is a twodimensional model, and the heights of the legs are not equal generally in different angles of the driving link in a complex linkage mechanism such as the Jansen linkage mechanism in this case $[13,38]$. And it is not general to contact the ground by three legs. Hence, the condition of contacting the ground by two legs consistently can be approximated.

The second approximation condition is that one leg does not slip. Such state represents walking with the highest efficiency. Referring to [10], we know that the Jansen linkage mechanism is able to walk with high efficiency in general. Thus, this condition can also be approximated.

For the first approximation condition, contacting with the ground by two legs can be regarded as constraining the toes on the ground (i.e., on the $x$-axis). For the second approximation condition, one leg not slipping can be regarded as the toe not having the speed in $x$ direction. Since these two constraints can be described as the position constraints, they can be treated as the holonomic constraints. The new constraint matrix is defined as follows:

$$
\begin{aligned}
\mathbf{C}_{a} & =\left[\begin{array}{lll}
\mathbf{C}_{a x}^{T} & \mathbf{C}_{a 1}^{T} & \mathbf{C}_{a 2}^{T}
\end{array}\right]^{T}, \\
\mathbf{C}_{a x} & =\frac{\partial\left(x_{i j 8}+h_{i j 8} \cos \left(\theta_{i j 8}-\gamma_{i j 8}\right)\right)}{\partial \mathbf{x}_{p}}, \\
\mathbf{C}_{a 1} & =\frac{\partial t_{r j}}{\partial \mathbf{x}_{p}}, \quad\left(t_{r j} \leq t_{l j}\right), \\
\mathbf{C}_{a 2} & =\frac{\partial t_{l j}}{\partial \mathbf{x}_{p}}, \quad\left(t_{l j}<t_{l j}\right),
\end{aligned}
$$

where $t_{i j}=y_{i j 8}+h_{i j 8} \sin \left(\theta_{i j 8}-\gamma_{i j 8}\right)$. Using this constraint matrix, (24) is expanded as

$$
\mathbf{M} \ddot{\mathbf{x}}_{p}=\mathbf{h}+\left[\begin{array}{l}
\mathbf{C} \\
\mathbf{C}_{a}
\end{array}\right]^{T}\left[\begin{array}{l}
\lambda \\
\lambda_{a}
\end{array}\right],
$$

which can be projected to the tangent speed space by using the orthogonal complement matrix $\mathbf{D}$. Thus we have the motion equation including contact forces on the plane:

$$
\mathbf{D}^{T} \mathbf{M D} \ddot{\mathbf{q}}+\mathbf{D}^{T} \mathbf{M} \dot{\mathbf{D}} \dot{\mathbf{q}}=\mathbf{D}^{T} \mathbf{h}+\mathbf{D}^{T} \mathbf{C}_{a}^{T} \boldsymbol{\lambda}_{a} .
$$

4.2. Transformation of Dynamic Model. Due to the presence of Lagrange's multiplier $\lambda_{a}$, model (39) which represents the constraint forces occurring on each constraint condition can not be utilized directly to the controller. To do that, (39) needs to be transformed into a usable form for the controller.

Firstly, the constraint forces of the system are obtained using the projection method $[39,40]$ :

$$
\lambda=\left(\mathbf{C M}^{-1} \mathbf{C}^{T}\right)^{-1} \mathbf{C}\left(\dot{\mathbf{D}} \dot{\mathbf{q}}-\mathbf{M}^{-1} \mathbf{h}\right) .
$$

Hence, the constraint forces of the dynamic model (29) can be represented as

$$
\left[\begin{array}{l}
\lambda \\
\lambda_{a}
\end{array}\right]=\left(\widehat{\mathbf{C}} \mathbf{M}^{-1} \widehat{\mathbf{C}}^{T}\right)^{-1} \widehat{\mathbf{C}}\left(\dot{\mathbf{D}} \dot{\mathbf{q}}-\mathbf{M}^{-1} \mathbf{h}\right),
$$

where

$$
\widehat{\mathbf{C}}=\left[\begin{array}{c}
\mathbf{C} \\
\mathbf{C}_{a}
\end{array}\right] .
$$

In the second step, the generalized force matrix $\mathbf{h}$ is expressed as the sum of three terms:

$$
\mathbf{h}=\mathbf{E} u+\mathbf{F} \dot{\mathbf{q}}+\mathbf{G} g,
$$

where $u$ is the input torque, and

$$
\begin{aligned}
& \mathbf{E}=\frac{\partial \mathbf{h}}{\partial u}=\mathbf{D}_{s}^{T}\left[\begin{array}{lllll}
\mathbf{E}_{g}^{T} & \mathbf{E}_{r f}^{T} & \mathbf{E}_{r b}^{T} & \mathbf{E}_{l f}^{T} & \mathbf{E}_{l b}^{T}
\end{array}\right]^{T}, \\
& \mathbf{F}=\frac{\partial(\mathbf{h}-\mathbf{E} u)}{\partial \dot{\mathbf{q}}}=\mathbf{D}_{s}^{T}\left(\mathbf{F}_{1}-\mathbf{F}_{2}\right) \\
& =\mathbf{D}_{s}^{T}\left(\operatorname{diag}\left(\mathbf{F}_{i j}\right)-\mathbf{M}_{s} \operatorname{diag}\left(\dot{\mathbf{D}}_{i j}\right)\right) \mathbf{D}, \\
& \mathbf{G}=\frac{\partial(\mathbf{h}-\mathbf{E} u-\mathbf{F} \dot{\mathbf{q}})}{\partial g} \\
& =\mathbf{D}_{s}^{T}\left[\begin{array}{lllll}
\mathbf{G}_{g}^{T} & \mathbf{G}_{r f}^{T} & \mathbf{G}_{r b}^{T} & \mathbf{G}_{l f}^{T} & \mathbf{G}_{l b}^{T}
\end{array}\right]^{T},
\end{aligned}
$$

with the following components:

$$
\begin{aligned}
& \mathbf{D}_{s}=\operatorname{diag}\left(\mathbf{D}_{g}, \mathbf{D}_{r f}, \mathbf{D}_{r b}, \mathbf{D}_{l f}, \mathbf{D}_{l b}\right), \\
& \mathbf{M}_{s}=\operatorname{diag}\left(\mathbf{M}_{g}, \mathbf{M}_{r f}, \mathbf{M}_{r b}, \mathbf{M}_{l f}, \mathbf{M}_{l f}\right), \\
& \mathbf{E}_{i j}=\frac{\partial \mathbf{h}_{i j}}{\partial u}, \\
& \mathbf{F}_{i j}=\frac{\partial\left(\mathbf{h}_{i j}-\mathbf{E}_{i j} u\right)}{\partial \dot{\mathbf{q}}_{i j}}, \\
& \mathbf{G}_{i j}=\frac{\partial\left(\mathbf{h}_{i j}-\mathbf{E}_{i j} u-\mathbf{F}_{i j} \dot{\mathbf{q}}_{i j}\right)}{\partial g} .
\end{aligned}
$$


Substituting the above terms into (39) and (41) and further simplification generate the following model for the controller:

$$
\begin{array}{rl}
\mathbf{D}^{T} & \mathbf{M D} \ddot{\mathbf{q}}+\mathbf{D}^{T}(\mathbf{M} \dot{\mathbf{D}}-\mathbf{F}-\boldsymbol{\alpha}) \dot{\mathbf{q}}-\mathbf{D}^{T}(\mathbf{G}+\boldsymbol{\gamma}) g \\
\quad=\mathbf{D}^{T}(\mathbf{E}+\boldsymbol{\beta}) u,
\end{array}
$$

where

$$
\begin{aligned}
& \boldsymbol{\alpha}=\widehat{\mathbf{C}}^{T}\left(\widehat{\mathbf{C}} \mathbf{M}^{-1} \widehat{\mathbf{C}}^{T}\right)^{-1} \widehat{\mathbf{C}}\left(\dot{\mathbf{D}}-\mathbf{M}^{-1} \mathbf{F}\right), \\
& \boldsymbol{\beta}=-\widehat{\mathbf{C}}^{T}\left(\widehat{\mathbf{C}} \mathbf{M}^{-1} \widehat{\mathbf{C}}^{T}\right)^{-1} \widehat{\mathbf{C}} \mathbf{M}^{-1} \mathbf{E}, \\
& \boldsymbol{\gamma}=-\widehat{\mathbf{C}}^{T}\left(\widehat{\mathbf{C}} \mathbf{M}^{-1} \widehat{\mathbf{C}}^{T}\right)^{-1} \widehat{\mathbf{C}} \mathbf{M}^{-1} \mathbf{G} .
\end{aligned}
$$

4.3. Control System Based on Energy Control. The control system is designed based on the dynamic model and the energy control which was proposed by Åström et al. as a control method focusing on the energy of the system [4143]. The energy-based control is better than state space representation for controlling complicated mechanisms, such as the quadruped robot with Jansen linkage mechanisms in this case. To realize the position control using energy approach, a potential field [44-47] is introduced. As for the potential field, the target position is designed as the point where the potential is the lowest. Hence, the process of motion control is the process that the energy of the robot is converging to the lowest potential, namely, the target position. We define the potential field as

$$
E_{p}=k_{p}\left(x_{l}-x_{r}\right)^{2}
$$

where the current position of the robot is defined as $x_{i} ; x_{r}$ represents the target position and is defined as $x_{r}=0 ; k_{p}$ is the gradient of the potential field. The kinetic energy of the system is defined as

$$
E_{k}=\frac{1}{2} \dot{\mathbf{x}}_{p}^{T} \mathbf{M}_{s} \dot{\mathbf{x}}_{p}
$$

Combining (48) and (49), the total energy $E$ of the system is

$$
E=E_{k}+E_{p} .
$$

Then we design a Lyapunov function

$$
V=\frac{1}{2}\left(E-E_{r}\right)^{2},
$$

where $E_{r}$ is the target energy, that is, $E_{r}=0$. Solving the differential of (51), we have

$$
\dot{V}=E \dot{E}=\left(E_{k}+E_{p}\right)\left(\dot{E}_{k}+\dot{E}_{p}\right),
$$

where

$$
\begin{aligned}
\dot{E}_{p} & =\frac{d}{d t} k_{p}\left(x_{\iota}-x_{r}\right)^{2}=2 k_{p} x_{\iota} \dot{x}_{\iota} \\
& =2 k_{p} \dot{\mathbf{q}}^{T}\left[0, x_{\iota}, 0,0\right]^{T}, \\
\dot{E}_{k} & =\frac{d}{d t} \frac{1}{2} \dot{\mathbf{x}}_{p}^{T} \mathbf{M}_{s} \dot{\mathbf{x}}_{p}=\dot{\mathbf{x}}_{p}^{T} \mathbf{M}_{s} \ddot{\mathbf{x}}_{p} .
\end{aligned}
$$

Considering $\dot{\mathbf{x}}_{p}=\mathbf{D}_{s} \mathbf{D} \dot{\mathbf{q}}$,

$$
\begin{aligned}
\dot{E}_{k} & =\dot{\mathbf{q}}^{T} \mathbf{D}^{T} \mathbf{D}_{s}^{T} \mathbf{M}_{s}\left(\mathbf{D}_{s} \mathbf{D} \ddot{\mathbf{q}}+\mathbf{D}_{s} \dot{\mathbf{D}} \dot{\mathbf{q}}+\dot{\mathbf{D}}_{s} \mathbf{D} \dot{\mathbf{q}}\right) \\
& =\dot{\mathbf{q}}^{T}\left(\mathbf{D}^{T} \mathbf{M D} \ddot{\mathbf{q}}+\mathbf{D}^{T} \mathbf{M} \dot{\mathbf{D}} \dot{\mathbf{q}}+\mathbf{D}^{T} \mathbf{F}_{2} \dot{\mathbf{q}}\right) .
\end{aligned}
$$

Transforming (46) into

$$
\begin{array}{rl}
\mathbf{D}^{T} & \mathbf{M D} \ddot{\mathbf{q}}+\mathbf{D}^{T} \mathbf{M D} \dot{\mathbf{q}}+\mathbf{D}^{T} \mathbf{F}_{2} \dot{\mathbf{q}} \\
\quad= & \mathbf{D}^{T}(\mathbf{E}+\boldsymbol{\beta}) u+\mathbf{D}^{T}\left(\mathbf{F}_{1}+\boldsymbol{\alpha}\right) \dot{\mathbf{q}}+\mathbf{D}^{T}(\mathbf{G}+\gamma) g
\end{array}
$$

and then substituting it into (55) yield

$$
\begin{aligned}
\dot{E}_{k} & =\dot{\mathbf{q}}^{T}\left(\mathbf{D}^{T}(\mathbf{E}+\boldsymbol{\beta}) u+\mathbf{D}^{T}\left(\mathbf{F}_{1}+\boldsymbol{\alpha}\right) \dot{\mathbf{q}}\right. \\
& \left.+\mathbf{D}^{T}(\mathbf{G}+\boldsymbol{\gamma}) g\right) .
\end{aligned}
$$

Substituting (53) and (57) into (52), the differential of the Lyapunov function can be represented as

$$
\begin{aligned}
\dot{V} & =\left(E_{k}+E_{p}\right) \dot{\mathbf{q}}^{T}\left(\mathbf{D}^{T}(\mathbf{E}+\boldsymbol{\beta}) u+\mathbf{D}^{T}\left(\mathbf{F}_{1}+\boldsymbol{\alpha}\right) \dot{\mathbf{q}}\right. \\
& \left.+\mathbf{D}^{T}(\mathbf{G}+\boldsymbol{\gamma}) g+2 k_{p}\left[0, x_{\imath}, 0,0\right]^{T}\right) .
\end{aligned}
$$

To ensure the Lyapunov stability (namely, to satisfy $\dot{V} \leq 0$ ), we choose the input torque as

$$
\begin{aligned}
u= & -\left(\mathbf{D}^{T}(\mathbf{E}+\boldsymbol{\beta})\right)^{\dagger}\left(k\left(E_{k}+E_{p}\right) \dot{\mathbf{q}}+\mathbf{D}^{T}(\mathbf{G}+\boldsymbol{\gamma}) g\right. \\
& \left.+2 k_{p}\left[0, x_{\iota}, 0,0\right]^{T}\right),
\end{aligned}
$$

where $\uparrow$ denotes the operator of pseudo inverse; $k$ denotes the tuning parameter and satisfies $k>0$.

Finally, substituting (59) into (58), we have

$$
\begin{aligned}
\dot{V} & =\left(E_{k}+E_{p}\right) \dot{\mathbf{q}}^{T}\left(-k\left(E_{k}+E_{p}\right) \dot{\mathbf{q}}-\mathbf{D}^{T}(\mathbf{G}+\gamma) g\right. \\
& -2 k_{p}\left[0, x_{\iota}, 0,0\right]^{T}+\mathbf{D}^{T}\left(\mathbf{F}_{1}+\boldsymbol{\alpha}\right) \dot{\mathbf{q}} \\
& \left.+\mathbf{D}^{T}(\mathbf{G}+\gamma) g+2 k_{p}\left[0, x_{\iota}, 0,0\right]^{T}\right)=-k\left(E_{k}\right. \\
& \left.+E_{p}\right)^{2} \dot{\mathbf{q}}^{T} \dot{\mathbf{q}}+\left(E_{k}+E_{p}\right) \dot{\mathbf{q}}^{T} \mathbf{D}^{T}\left(\mathbf{F}_{1}+\boldsymbol{\alpha}\right) \dot{\mathbf{q}}
\end{aligned}
$$

where $\dot{\mathbf{q}}^{T} \mathbf{D}^{T}\left(\mathbf{F}_{1}+\boldsymbol{\alpha}\right) \dot{\mathbf{q}} \leq 0$ because $\dot{\mathbf{q}}^{T} \mathbf{D}^{T}\left(\mathbf{F}_{1}+\boldsymbol{\alpha}\right) \dot{\mathbf{q}}$ represents the viscous friction forces. According to (48) and (49), $\left(E_{k}+\right.$ $\left.E_{p}\right) \geq 0$. Therefore,

$$
\dot{V} \leq 0
$$

is proven by using (59) as the input torque. In addition, from (51) and (52), it is obvious that

$$
\begin{aligned}
& V(0)=0, \\
& \dot{V}(0)=0,
\end{aligned}
$$

and an equilibrium point of the Lyapunov function is the case of $\mathbf{x}_{p}=0$; therefore the system is proven to be Lyapunov stable [48]. Furthermore, the system is asymptotically stable as long as $\dot{\mathbf{q}} \neq 0$. Therefore, the position of the robot can be converged to the target position by using the energy-based control strategy. 


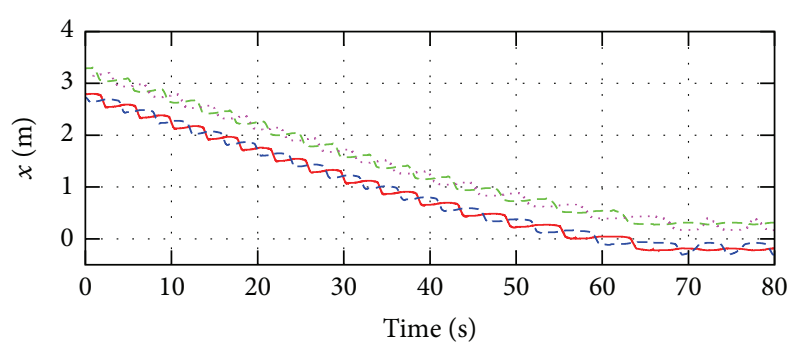

(a)

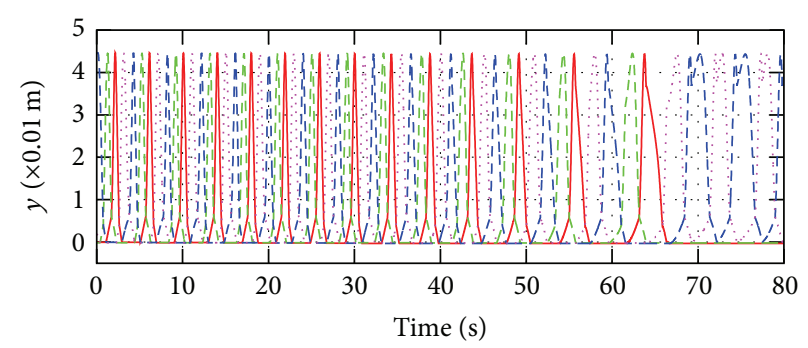

(b)

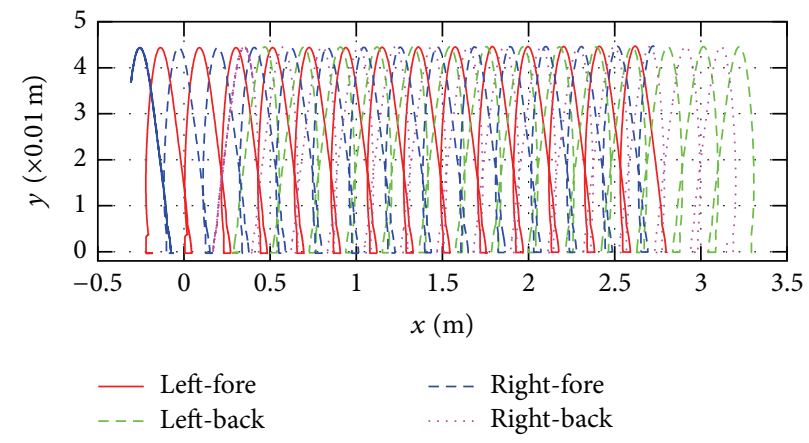

(c)

FIGURE 6: Time evolutions of $x$-coordinate (a) and $y$-coordinate (b) of the robot's toes and the trajectory of the feet (c).

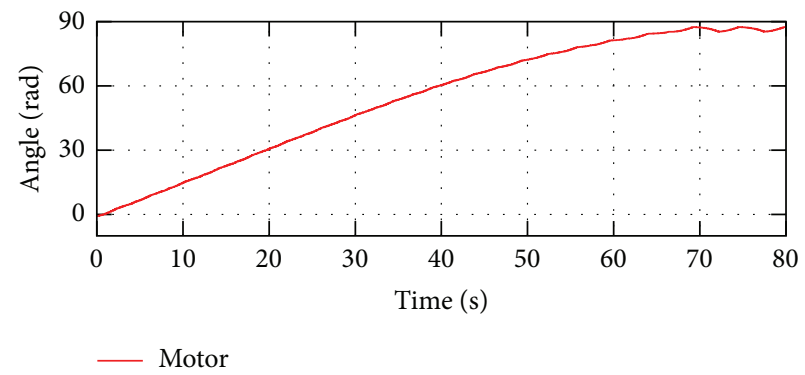

(a)

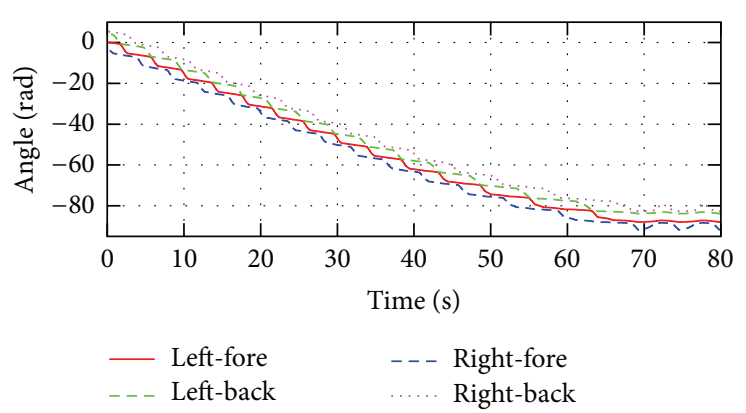

(b)

FIgURE 7: Time evolution of the angles of the driving gear $\theta_{m}$ (a) and driven gears $\theta_{i j}$ (b).

\section{Numerical Simulations}

Effectiveness of the designed position control system utilizing the approximate model is verified by numerical simulations which are performed by MaTX with Visual C++ 2005 version 5.3.37 [49]. The parameters used in simulations are those shown in Tables 1 and 4 . The simulation time is 80 seconds, and the sampling interval is 0.01 second. The initial state of the robot is halted on the plane at position $x_{l}=3 \mathrm{~m}$ and the Jansen link mechanism is with $\theta_{m}=-\pi / 4 \mathrm{rad}$. In addition, the gradient of the potential field $k_{p}=1$ and the tuning parameter $k=0.3$. The coefficient values of kinetic and viscous frictions are given as $\mu_{d}=0.1$ and $\mu_{v}=0.1$. The numerical simulation results are shown as follows.

From Figures 6(a) and 6(b), it is confirmed that the robot moves with toes slipping. Figure 6(c) shows that the noncompletely elastic collisions occur at the toes of the robot (i.e., $\dot{t}_{i j}=0$ ) when the toes collide with the ground (i.e., $t_{i j}=0$ ) and height of the toes is greater than or equal to 0 . In addition, in case the toes depart from the ground, the negative floor reaction force (i.e., the constraint force of $\lambda_{i j}<0$ ) is not generated because the position of the toe varies smoothly. Therefore, the dynamic models given in Sections 2 and 3 represent the dynamics of the robot shown in Figure 1 in twodimensional surface including the contact and collision with the ground. Meanwhile, the rotation angle of the driving gear is increasing from zero to $90^{\circ}$, and those of the four driven gears are varying smoothly to $-80^{\circ} \sim-90^{\circ}$ approximately (Figure 7).

From Figures 8 and 9, it is confirmed that the position of the robot can be converged to $x=0$ by the designed position control system. Meanwhile Figure 10 shows that the energy of the system converges to 0 . In addition, since the value of the Lyapunov function converges to 0 and its 


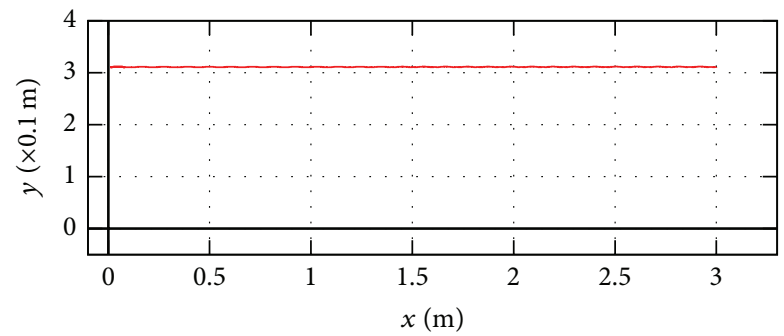

Figure 8: Trajectory of robot's position.

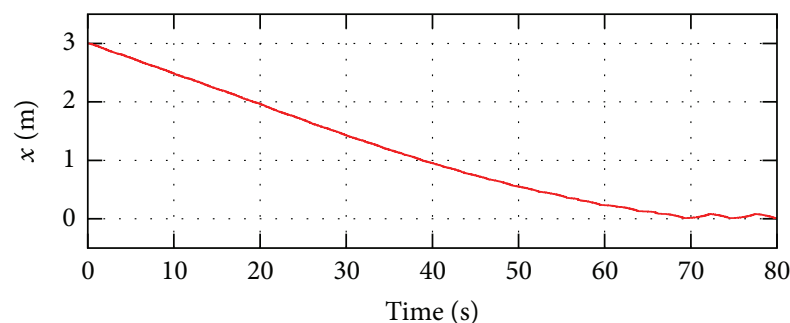

FIGURE 9: Time evolution of $x$-coordinate of the robot.

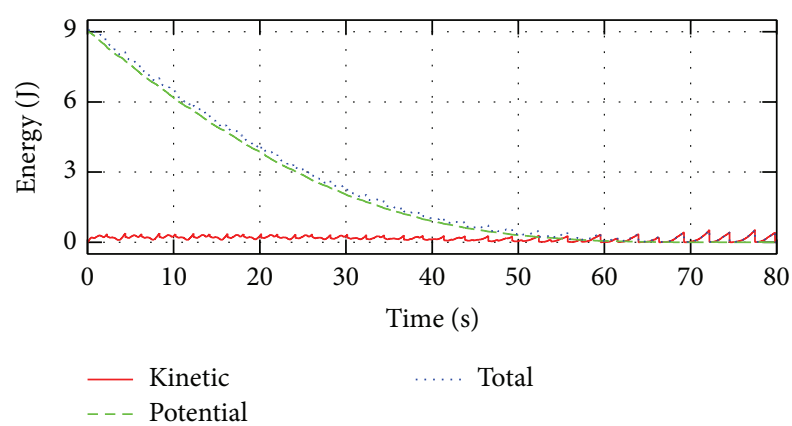

Figure 10: Time evolutions of the kinetic energy $E_{k}$ and the potential energy $E_{p}$.

differential is also less than 0 as shown in Figure 11, it is confirmed that the input torque shown in Figure 12 satisfies Lyapunov's stability theory. Therefore, the position of the quadruped robot with the Jansen linkage mechanism can be controlled by applying the position control system based on the energy control. Figure 13 shows a sequence of the snapshots of walking towards the target position based on the energy-based position control in 80 seconds.

\section{Conclusion}

This paper contributes to model and analyze the dynamics of the four-legged robots based on Theo Jansen linkage mechanisms and driven by only one input using the projection method. The free-fall model of the whole system is built through integrating the models of the Jansen linkages and the corresponding gear system. Based on that, the reaction

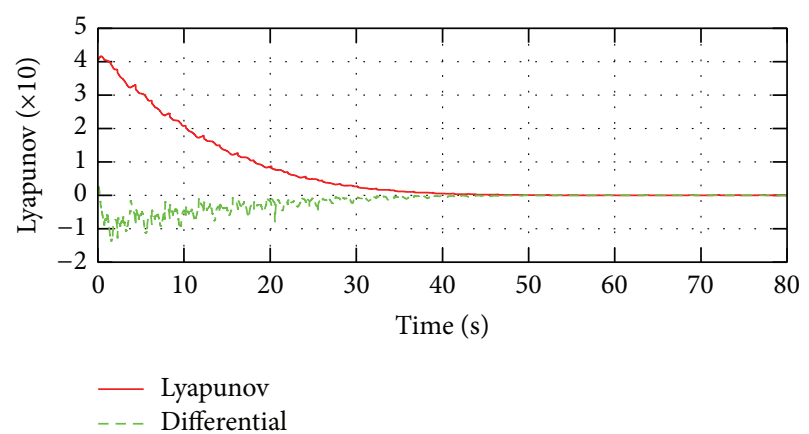

FIGURE 11: Time evolutions of the values of Lyapunov's function $V$ and its differential $\dot{V}$.

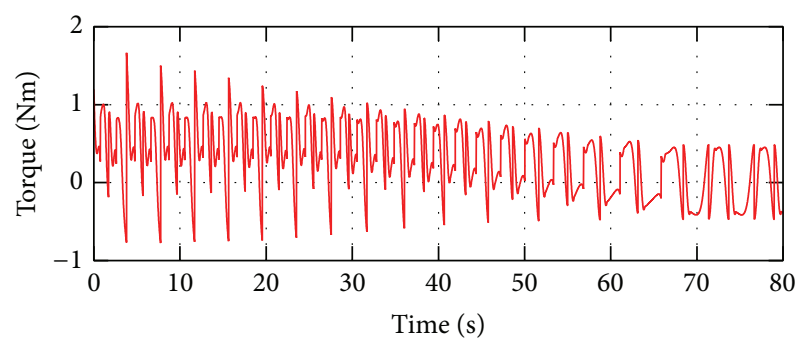

Figure 12: Time evolution of the input torque $u$.

force and friction are taken into account to derive the plant model. The energy control system utilizing the potential field is designed to realize the position control of the Jansen walking robot. Based on the Lyapunov stability theory, this controller is proven to be able to converge by choosing appropriate input and its effectiveness is verified through numerical simulations. This research sets a theoretical basis for further investigation, optimization, or extension of legged systems based on Theo Jansen linkage mechanisms.

\section{Conflict of Interests}

The authors declare that there is no conflict of interests regarding the publication of this paper. 

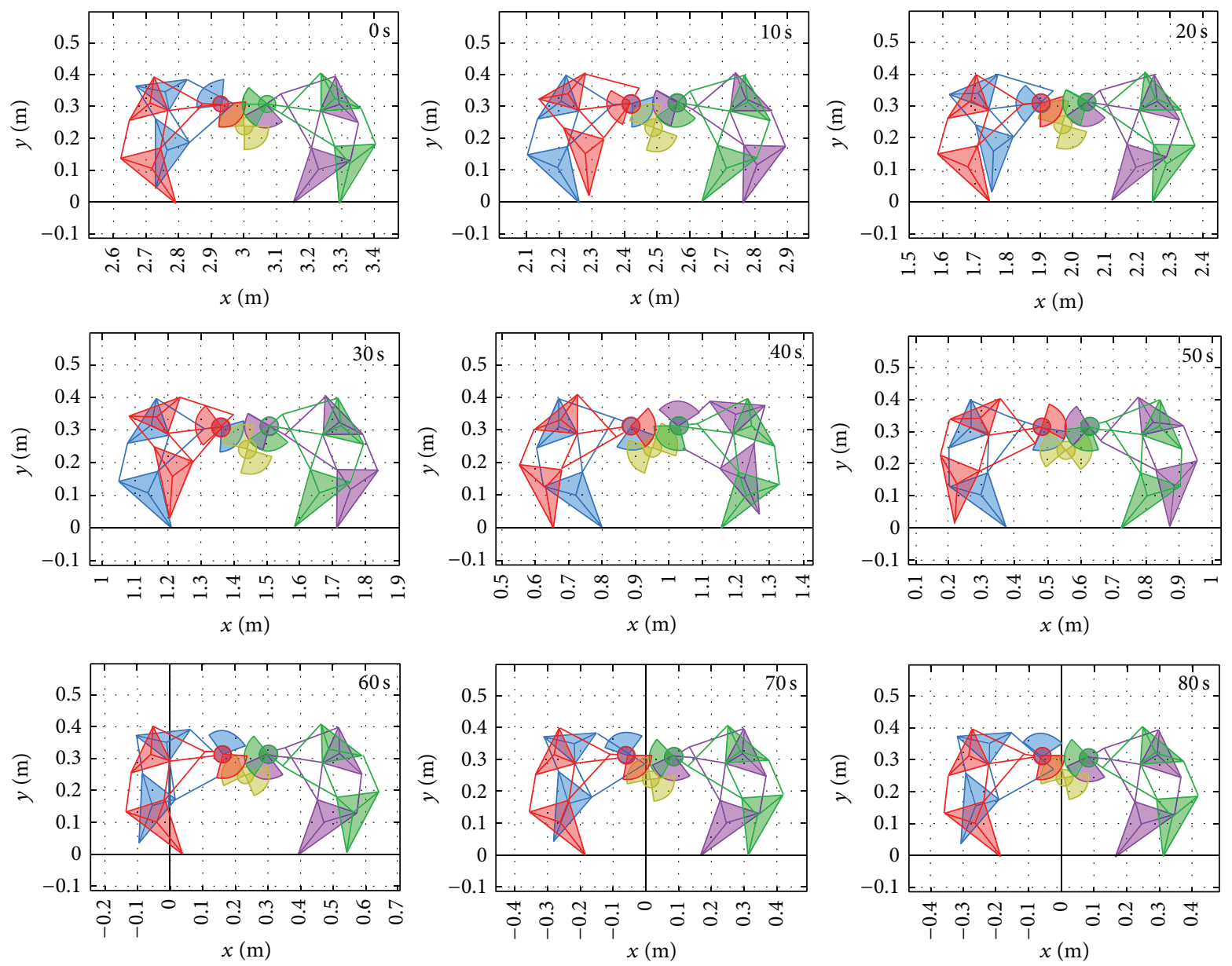

FIGURE 13: The snapshots of walking towards the target position based on the energy-based position control.

\section{Acknowledgment}

This work was fully supported by the SUTD-MIT International Design Centre, Singapore, under Grants IDG31200110 and IDD41200105.

\section{References}

[1] H. Kazemi, V. J. Majd, and M. M. Moghaddam, "Modeling and robust backstepping control of an underactuated quadruped robot in bounding motion," Robotica, vol. 31, no. 3, pp. 423-439, 2013.

[2] M. F. Silva and J. A. T. MacHado, "Kinematic and dynamic performance analysis of artificial legged systems," Robotica, vol. 26, no. 1, pp. 19-39, 2008.

[3] C. A. Acosta-Calderon, R. E. Mohan, C. Zhou, L. Hu, P. K. Yue, and H. Hu, "A modular architecture for humanoid soccer robots with distributed behavior control," International Journal of Humanoid Robotics, vol. 5, no. 3, pp. 397-416, 2008.

[4] S. Bartsch, T. Birnschein, M. Römmermann, J. Hilljegerdes, D. Kühn, and F. Kirchner, "Development of the six-legged walking and climbing robot SpaceClimber," Journal of Field Robotics, vol. 29, no. 3, pp. 506-532, 2012.

[5] J. Estremera, J. A. Cobano, and P. G. de Santos, "Continuous free-crab gaits for hexapod robots on a natural terrain with forbidden zones: an application to humanitarian demining," Robotics and Autonomous Systems, vol. 58, no. 5, pp. 700-711, 2010.

[6] F. L. Moro, A. Spröwitz, A. Tuleu et al., "Horse-like walking, trotting, and galloping derived from kinematic Motion Primitives (kMPs) and their application to walk/trot transitions in a compliant quadruped robot," Biological Cybernetics, vol. 107, no. 3, pp. 309-320, 2013.

[7] D. Sanz-Merodio, E. Garcia, and P. Gonzalez-de-Santos, "Analyzing energy-efficient configurations in hexapod robots for demining applications," Industrial Robot, vol. 39, no. 4, pp. 357364, 2012.

[8] P. G. de Santos, E. Garcia, R. Ponticelli, and M. Armada, "Minimizing energy consumption in hexapod robots," Advanced Robotics, vol. 23, no. 6, pp. 681-704, 2009.

[9] S. S. Roy and D. K. Pratihar, "Dynamic modeling, stability and energy consumption analysis of a realistic six-legged walking robot," Robotics and Computer-Integrated Manufacturing, vol. 29, no. 2, pp. 400-416, 2013.

[10] T. Jansen, The Great Pretender, Nai010 Publishers, 2007.

[11] K. Komoda and H. Wagatsuma, "A proposal of the extended mechanism for theo jansen linkage to modify the walking elliptic orbit and a study of cyclic base function," in Proceedings of the 7th Annual Dynamic Walking Conference (DWC '12), May 2012. 
[12] F. Moldovan and V. Dolga, "Analysis of Jansen walking mechanism using CAD," Solid State Phenomena, vol. 166-167, pp. 297$302,2010$.

[13] A. J. Ingram, A new type of walking machine [Ph.D. thesis], University of Johannesburg, 2006.

[14] D. Giesbrecht and C. Q. Wu, "Dynamics of legged walking mechanism 'wind beast", in Proceedings of the Dynamic Walking Conference, 2009.

[15] W. Khalil and S. Guegan, "Inverse and direct dynamic modeling of Gough-Stewart robots," IEEE Transactions on Robotics and Automation, vol. 20, no. 4, pp. 754-762, 2004.

[16] X. Wang and J. K. Mills, "Dynamic modeling of a flexiblelink planar parallel platform using a substructuring approach," Mechanism and Machine Theory, vol. 41, no. 6, pp. 671-687, 2006.

[17] S. S. Mahil, "On the application of Lagrange's method to the description of dyanmic system," IEEE Transactions on Systems, Man and Cybernetics, vol. 12, no. 6, pp. 877-889, 1982.

[18] K. Arczewski and W. Blajer, "A unified approach to the modelling of holonomic and nonholonomic mechanical systems," Mathematical Modelling of Systems, vol. 2, no. 3, pp. 157-174, 1996.

[19] W. Blajer, "A geometrical interpretation and uniform matrix formulation of multibody system dynamics," Zeitschrift für Angewandte Mathematik und Mechanik, vol. 81, no. 4, pp. 247259, 2001.

[20] H. Ohsaki, M. Iwase, and S. Hatakeyama, "A consideration of nonlinear system modeling using the projection method," in Proceedings of the Society of Instrument and Control Engineers Annual Conference (SICE '07), pp. 1915-1920, September 2007.

[21] D. Studer, "My four legs walking machine," http://www.armure .ch/WALKING.htm.

[22] H. Ohsaki, M. Iwase, T. Sadahiro, and S. Hatakeyama, "A consideration of human-unicycle model for unicycle operation analysis based on moment balancing point," in Proceedings of the IEEE International Conference on Systems, Man and Cybernetics (SMC '09), pp. 2468-2473, October 2009.

[23] Y. Itoh, K. Higashi, and M. Iwase, "UKF-based estimation of indicated torque for IC engines utilizing nonlinear two-inertia model," in Proceedings of the 51st IEEE Conference on Decision and Control (CDC '12), pp. 4077-4082, December 2012.

[24] S. Nansai, N. Rojas, M. R. Elara, and R. Sosa, "Exploration of adaptive gait patterns with a reconfigurable linkage mechanism," in Proceedings of the 26th IEEE/RSJ International Conference on Intelligent Robots and Systems (IROS '13), pp. 4661-4668, November 2013.

[25] C. Canudas de Wit, H. Olsson, K. J. Åström, and P. Lischinsky, "A new model for control of systems with friction," IEEE Transactions on Automatic Control, vol. 40, no. 3, pp. 419-425, 1995.

[26] I. Virgala and M. Kelemen, "Experimental friction identification of a DC motor," International Journal of Mechanics and Applications, vol. 3, no. 1, pp. 26-30, 2013.

[27] D. Mundo, G. Gatti, and D. B. Dooner, "Optimized five-bar linkages with non-circular gears for exact path generation," Mechanism and Machine Theory, vol. 44, no. 4, pp. 751-760, 2009.

[28] E. Ottaviano, D. Mundo, G. A. Danieli, and M. Ceccarelli, "Numerical and experimental analysis of non-circular gears and cam-follower systems as function generators," Mechanism and Machine Theory, vol. 43, no. 8, pp. 996-1008, 2008.
[29] D. Mundo, "Geometric design of a planetary gear train with non-circular gears," Mechanism and Machine Theory, vol. 41, no. 4, pp. 456-472, 2006.

[30] S. Nansai, M. R. Elara, and M. Iwase, "Dynamic analysis and modeling of Jansen mechanism," Procedia Engineering, vol. 64, pp. 1562-1571, 2013.

[31] H. Ohta, M. Yamakita, and K. Furuta, "From passive to active dynamic walking," International Journal of Robust and Nonlinear Control, vol. 11, no. 3, pp. 287-303, 2001.

[32] C. Canudas de Wit and P. Lischinsky, "Adaptive friction compensation with partially known dynamic friction model," International Journal of Adaptive Control and Signal Processing, vol. 11, no. 1, pp. 65-80, 1997.

[33] J. Swevers, F. Al-Bender, C. G. Ganseman, and T. Prajogo, "An integrated friction model structure with improved presliding behavior for accurate friction compensation," IEEE Transactions on Automatic Control, vol. 45, no. 4, pp. 675-686, 2000.

[34] H. Olsson, K. J. Åström, C. C. de Wit, M. Gäfvert, and P. Lischinsky, "Friction models and friction compensation," European Journal of Control, vol. 4, no. 3, pp. 176-195, 1998.

[35] V. Lampaert, J. Swevers, and F. Al-Bender, "Modification of the Leuven integrated friction model structure," IEEE Transactions on Automatic Control, vol. 47, no. 4, pp. 683-687, 2002.

[36] A. Ohata, A. Sugiki, Y. Ohta, S. Suzuki, and K. Furuta, "Engine modeling based on projection method and conservation laws," in Proceedings of the IEEE International Conference on Control Applications, vol. 2, pp. 1420-1424, IEEE, September 2004.

[37] S. Nansai, M. Iwase, and M. R. Elara, "Energy based position control of jansen walking robot," in Proceedings of the IEEE International Conference on Systems, Man, and Cybernetics, pp. 1241-1246, October 2013.

[38] F. Moldovan, V. Dolga, and C. Pop, "Kinetostatic analysis of an articulated walking mechanism," Mechanisms and Machine Science, vol. 3, pp. 103-110, 2012.

[39] K. Watanabe, M. Iwase, S. Hatakeyama, and T. Maruyama, "Control strategy for a snake-like robot based on constraint force and verification by experiment," in Proceedings of the IEEE/RSJ International Conference on Intelligent Robots and Systems (IROS '08), pp. 1618-1623, September 2008.

[40] K. Furuta, "Super mechano-systems: fusion of control and mechanism," in Proceedings of the 15th Triennial World Congress of IFAC, vol. 15, p. 1635, Barcelona, Spain, July 2002.

[41] K. J. Åström and K. Furuta, "Swinging up a pendulum by energy control," Automatica, vol. 36, no. 2, pp. 287-295, 2000.

[42] K. J. Åström, J. Aracil, and F. Gordillo, "A family of smooth controllers for swinging up a pendulum," Automatica, vol. 44, no. 7, pp. 1841-1848, 2008.

[43] J. Aracil, F. Gordillo, and K. J. Åström, "A new family of pumping-dumping smooth strategies for swinging up a pendulum," in Proceedings of the 16th IFAC World Congress, July 2005.

[44] Y. K. Hwang and N. Ahuja, "A potential field approach to path planning," IEEE Transactions on Robotics and Automation, vol. 8, no. 1, pp. 23-32, 1992.

[45] E. Rimon and D. E. Koditschek, "Exact robot navigation using artificial potential functions," IEEE Transactions on Robotics and Automation, vol. 8, no. 5, pp. 501-518, 1992.

[46] J. Barraquand, B. Langlois, and J. C. Latombe, "Numerical potential field techniques for robot path planning," IEEE Transactions on Systems, Man, and Cybernetics, vol. 22, no. 2, pp. 224241, 1992. 
[47] Y. Koren and J. Borenstein, "Potential field methods and their inherent limitations for mobile robot navigation," in Proceedings of the IEEE International Conference on Robotics and Automation, pp. 1398-1404, April 1991.

[48] R. M. Murray, Z. Li, and S. S. Sastry, A Mathematical Introduction to Robotic Manipulation, CRC Press, 1994.

[49] M. Koga, "MaTX/RtMaTX: a freeware for integrated CACSD," in Proceedings of the IEEE International Symposium on Computer Aided Control System Design, pp. 451-456, Kohala Coast, Hawaii, USA, August 1999. 

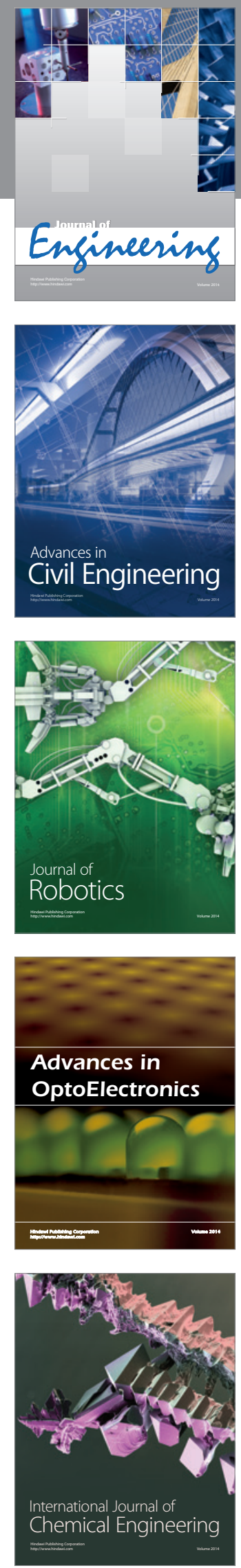

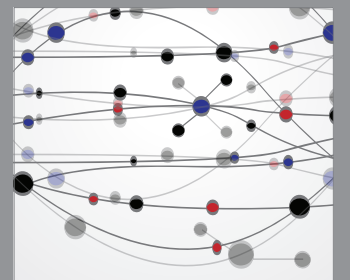

The Scientific World Journal
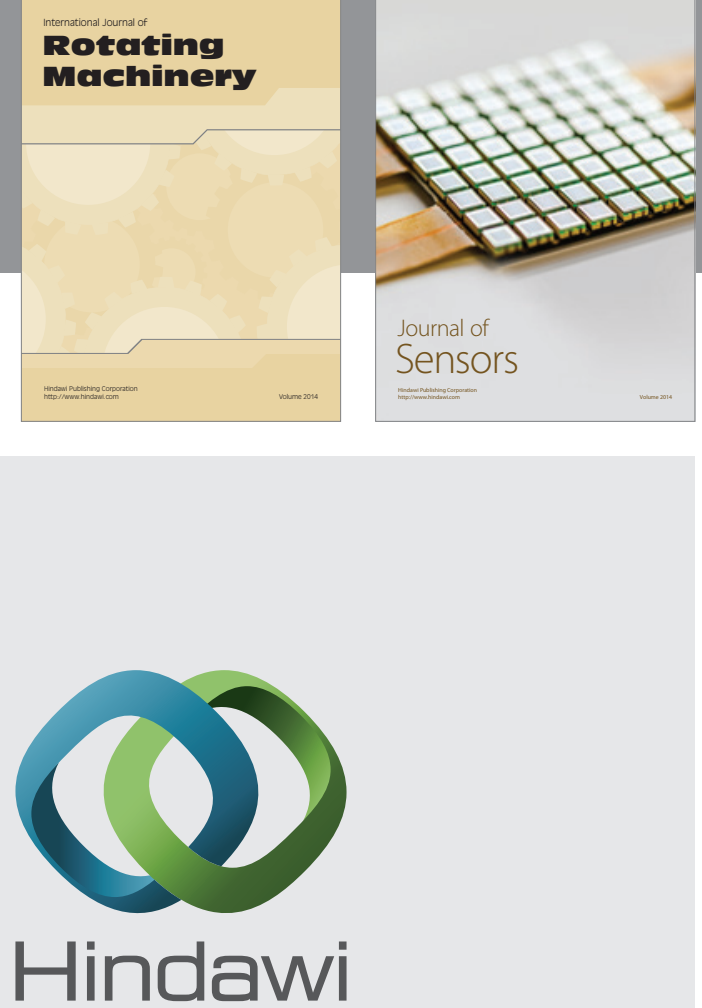

Submit your manuscripts at http://www.hindawi.com
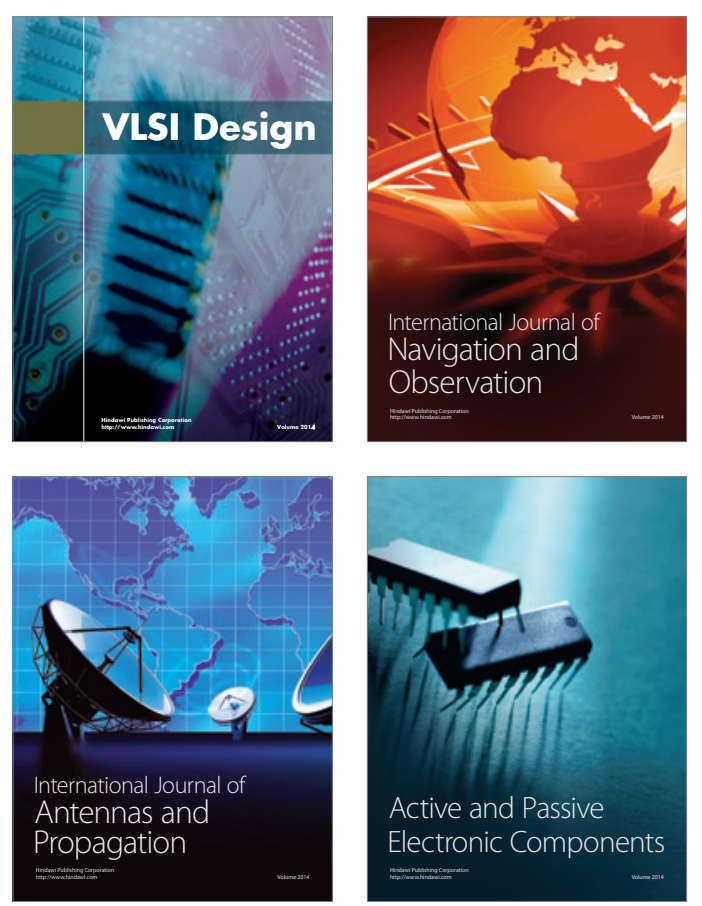
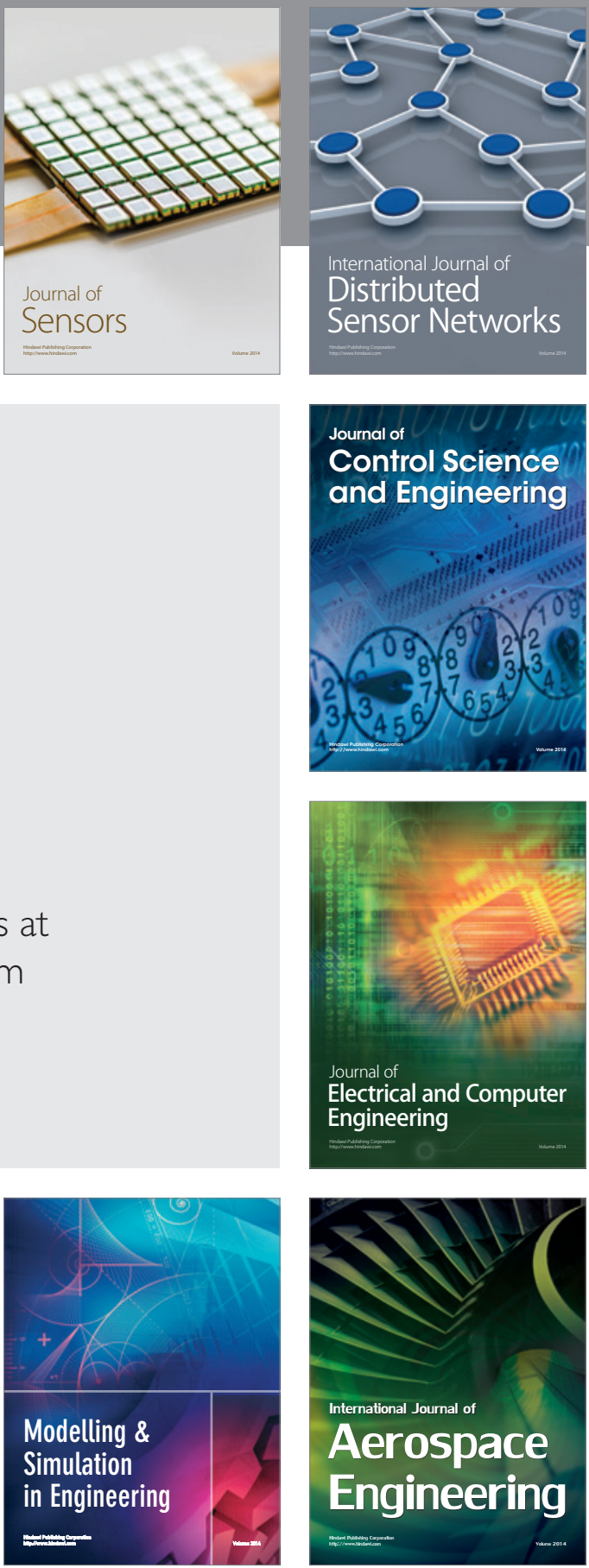

Journal of

Control Science

and Engineering
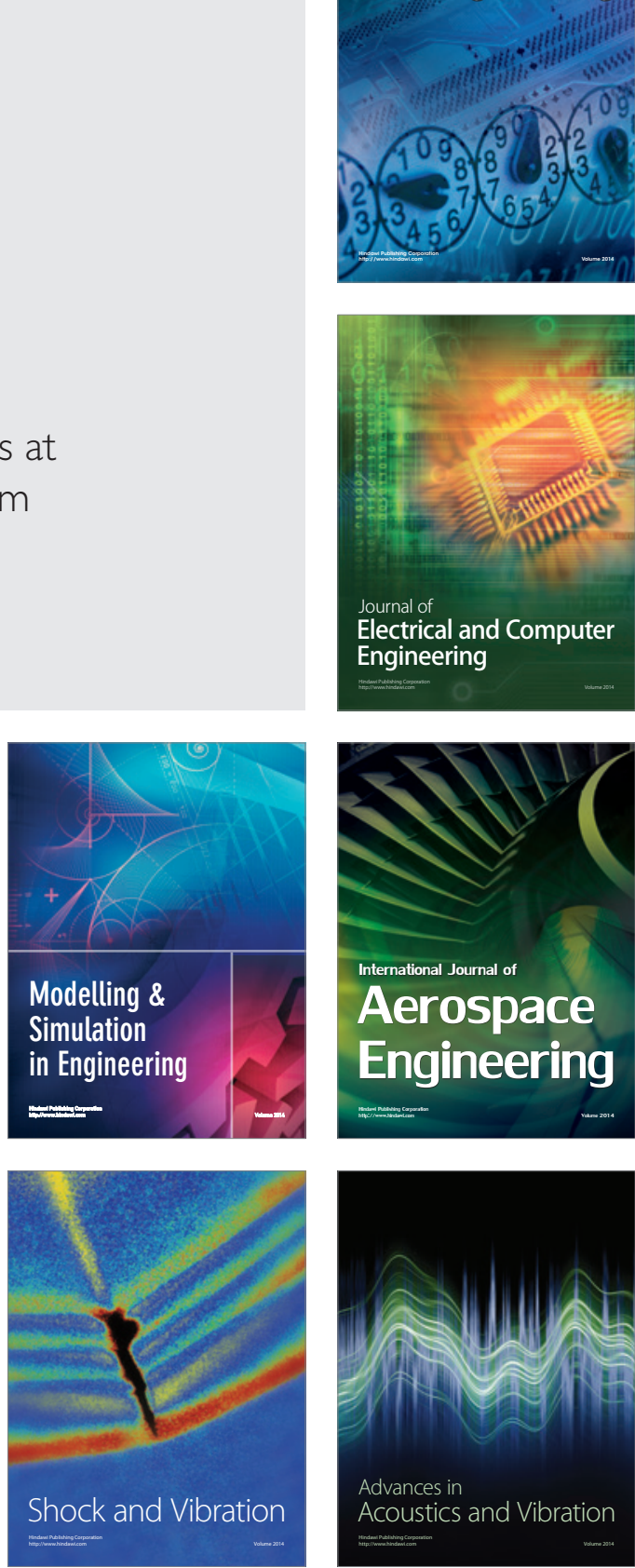